\title{
ÉTUDE DES POPULATIONS \\ D'AUSTROPOTAMOBIUS PALLIPES (CRUSTACEA, ASTACIDAE) DANS UN RUISSEAU FORESTIER DE NORMANDIE. I. STRUCTURES DÉMOGRAPHIQUES ET CROISSANCE : STABILITÉ ET VARIABILITÉ AU COURS DE SIX ANNÉES.
}

\author{
A. NEVEU \\ INRA, Laboratoire Ecologie Aquatique, UMR INRA-ENSAR, \\ 65, rue de St Brieuc, 35042 Rennes Cedex.

\section{RÉSUMÉ}

Les populations d'Austropotamobius pallipes ont été analysées sur deux sites d'un ruisseau forestier de Normandie, au cours de six années pour le site aval et de cinq années pour le site amont. Au début de chaque automne des échantillons ont été prélevés à l'aide de filets à mailles fines $(3 \mathrm{~mm})$, après avoir délogés les animaux en remuant le substrat. Comme cette technique est peu sélective les juvéniles de l'année sont capturés. Un codage différentiel par coupure des différentes parties de la queue a permis de suivre l'évolution des cohortes.

Des résultats acquis, il est possible de définir des classes d'âge et de calculer les tailles moyennes des individus de chaque classe pour suivre l'évolution de la croissance.

L'augmentation de la taille est régulière et suit un modèle linéaire avec le temps. La croissance est faible $(10,6$ à $18,8 \mathrm{~mm}$ par an), les individus au-delà de $90 \mathrm{~mm}$ sont très rares. La durée de vie est de 5 à 6 ans en aval, 4 à 5 ans en amont, la reproduction débutant la $3^{\text {eme }}$ année. La période de croissance est réduite aux mois d'été, elle est maximum la $2^{\text {ème }}$ et $3^{\text {ème }}$ années. La croissance en $2^{\text {éme }}$ et $3^{\text {ème }}$ années est inversement proportionnelle à celle acquise l'année précédente.

La croissance est plus forte en amont du ruisseau qu'en aval, avec une séparation plus nette des cohortes, mais il existe un étalement plus grand de celles-ci en relation avec une température plus élevée. La variabilité interannuelle de l'étalement des cohortes est plus faible en amont pour un taux de croissance plus variable.

La variabilité des tailles augmente avec l'âge ; elle se stabilise ensuite, alors que le taux de croissance se réduit. Au cours de l'étude la taille a tendance à augmenter et les différences intercohortes à se réduire, en relation avec des hivers plus doux.

Le sex-ratio est équilibré ; la croissance des mâles est plus élevée que celle des femelles en aval, alors qu'en amont la croissance des deux sexes est comparable.

Cette étude montre que dans un même ruisseau, des stations peu éloignées peuvent avoir des populations qui présentent des évolutions différentes, malgré un environnement a priori stabilisé par le couvert forestier. 
Austropotamobius pallipes paraît être très sensible aux variations thermiques et, par conséquent, l'étude à long terme de sa croissance pourrait être un bon indicateur des changements climatiques.

Mots-clés : Astacidae, Austropotamobius pallipes, structure des populations, croissance, variabilité spatio-temporelle, ruisseau, Normandie, France.

\section{STUDY OF AUSTROPOTAMOBIUS PALLIPES (CRUSTACEA, ASTACIDAE) POPULATIONS IN A FOREST BROOK IN NORMANDY. I. DEMOGRAPHIC STRUCTURES AND GROWTH : STABILITY AND VARIABILITY DURING SIX YEARS.}

\section{SUMMARY}

Austropotamobius pallipes populations were studied in two sites of a forest brook in Normandy (Western part of France), during six years (1990-1995) for A (downstream) and five years (1991-1995) for B (upstream). At the beginning of each October month crayfishes were sampled by hand nets with small mesh size $(3 \mathrm{~mm})$; as nets sampling technique is not very selective, many youngs of the year were caught. A marking technique, with cut of small pieces on tail board, allowed to follow temporal evolution in cohorts.

From obtained results it is possible to separate age classes and to estimate mean size by each classe in order to follow growth evolution through years.

Size increase is regular and follows a linear regression in the time. The growth is slow (10.6 to $18.8 \mathrm{~mm}$ per year), individuals above $90 \mathrm{~mm}$ are very rare. Life span is 5 to 6 years long downstream site, 4 to 5 years upstream site, reproductive activity begins only after three summers. Growth period is restricted to summer months and maximum during the second and third year. Growth rate at two and three years old is negatively correlated with this occurring the previous year.

Growth is better upstream site than downstream one with a more distinct cohort structure. But there were larger spreading cohorts in relation to higher thermal regime.

Variability of cohorts spreading between years is smaller in upstream site with a growth rate more variable. Size variability increases with age growth rate decreases. During the study crayfishes size shows a tendency to increase and differences between cohorts to decrease in relation to milder winters.

Sex-ratio is very close to one. Males growth is better than females one downstream, when growth is not different between the two sexes upstream.

This study shows that two sites not to far away in the same brook can have populations with different life histories, in spite of buffered surrounding conditions by canopy of an old forest. Austropotamobius pallipes seems be very sensitive to local variations and consequently long term studies of growth could be a good bioindicator of climate global change.

Key-words : Astacidae, Austropotamobius pallipes, population structure, growth, spacio temporal variability, brook, Normandy, France. 


\section{INTRODUCTION}

Au cours d'une prospection récente de nombreuses populations résiduelles d'écrevisses à pattes blanches Austropotamobius pallipes (Lereboullet, 1858) ont été détectées dans l'Ouest de la France, en particulier dans la zone du parc régional Normandie-Maine. Cette région est riche en zones forestières, constituées souvent de futaies anciennes et parcourues par de nombreux ruisseaux aux eaux de bonne qualité, favorables à la pérennité de cette espèce d'écrevisse dont les populations sont globalement en forte régression.

Une étude préliminaire (en 1989-1990) sur plusieurs populations a montré les difficultés d'analyse démographique de cette espèce, en particulier faute de moyens efficaces et non biaisés de capture, de marquage et de références squelettochronologiques indicatrices de l'âge et de l'histoire individuelle (NEVEU, 1996). Les données acquises ont permis de sélectionner certaines approches méthodologiques efficaces sur les petits ruisseaux et de déterminer la richesse de certains habitats. Aussi le nouvel objectif a-t-il été de suivre plus précisément une population relativement abondante, au niveau de sa structure démographique, mais surtout de son évolution à moyen terme au cours de six années de 1990 à 1995 pour le site aval et de cinq années de 1991 à 1995 pour le site amont.

Le premier problème à résoudre fut l'accès aux classes d'âges en l'absence de références sur les individus. De nombreuses méthodes statistiques existent pour l'extraction des divers composants d'une distribution polymodale, mais encore faut-il être sûr que les groupes définis ainsi correspondent à une réalité biologique. II faut adopter un compromis entre la précision statistique et la plausibilité biologique (Mc DONALD et PITCHER, 1979). L'application de ces méthodes aux écrevisses a été effective sur plusieurs espèces (FRANCE et al., 1991 ; ZEKHNINI et CHAISEMARTIN, 1991 ; MANKAMPA, 1995 ; NEVEU, 1996 ; ROQUEPLO, 1997). Mais l'approche statistique montre que certaines conditions sont difficiles à réaliser, et surtout non vérifiées par les auteurs, en particulier au niveau de la normalité des distributions ou au moins de leur concordance avec une loi de distribution classique (Mc DONALD et PITCHER, 1979). Par ailleurs ces auteurs montrent aussi la nécessité d'un échantillon minimum, au moins 200 individus pour 4 modes, soit environ 50 individus par classe d'âge, ce que confirme FRANCE et al. (1991) sur les écrevisses Orconectes virilis. Malgré tout, ces derniers soulignent la nécessité de contrôler par marquage in situ la réalité biologique, ou par élevage pour la croissance et le nombre de mues, tout en confirmant les remarques de DAVIES (1989) sur les méthodes graphiques classiques considérées comme subjectives mais relativement efficaces.

Dans les petits ruisseaux il est souvent très difficile d'obtenir une taille d'échantillon suffisante pour chaque classe d'âge, l'effort de pêche étant trop important. Par ailleurs la normalité des distributions peut être perturbée par la croissance différente des sexes, les mâles évoluant souvent plus vite que les femelles, ce qui augmente la taille de l'échantillon, s'il faut traiter indépendamment les mâles des femelles. Une autre perturbation est liée à l'impact des mues à un instant donné : sur un même ensemble d'individus, y compris une fratrie, une partie peut avoir effectué une mue de plus que leurs congénères et créer ainsi une bimodalité (ou polymodalité) passagère, créant un artefact dans l'approche statistique. A priori les mesures en période de mues intenses doivent être évitées. De même des irrégularités de croissance en liaison avec la qualité de l'habitat peuvent créer des différences au sein d'une même classe d'âge.

Le premier objectif de cette étude a été un suivi aussi précis que possible de l'évolution de différents groupes de taille par un marquage différentiel. A partir de ces données un découpage en classes d'âge est possible, ainsi qu'une analyse précise de l'évolution de la croissance moyenne des individus. Le deuxième objectif a été alors de suivre l'évolution de cette croissance sur plusieurs années, de détecter la variabilité et/ou 
la stabilité de certains paramètres démographiques en liaison avec un environnement a priori favorable et stable (vieilles forêts). Jusqu'à présent les études démographiques à moyen et long termes font défaut dans la littérature sur les écrevisses à pattes blanches, la plus longue ( 3 ans) étant celle de BREWIS et BOWLER (1982).

\section{MÉTHODES D'ÉTUDE}

\section{Localisation des sites}

Le choix des zones d'échantillonnage s'est effectué à partir des résultats de l'étude préliminaire de 1989-1990 dans la région Normandie. Le choix s'est porté sur le ruisseau du Vivier ( $0^{\circ} 31^{\prime} 00^{\prime \prime}$ Ouest, $48^{\circ} 35^{\prime} 32^{\prime \prime}$ Nord), affluent d'ordre 2 de l'Andainette en relation avec deux caractéristiques essentielles : une population assez dense $\left(6\right.$ à 12 ind $/ / \mathrm{m}^{2}$ hors juvéniles, NEVEU, 1996) et un milieu a priori stable et protégé par le rôle tampon de la forêt domaniale des Andaines couvrant tout le bassin versant.

Le ruisseau du Vivier est issu de 2 étangs forestiers, plus ou moins marécageux, sa longueur totale est de $3,4 \mathrm{~km}$, sa largeur est de 1 à $3 \mathrm{~m}$. Le site d'étude en aval (A), est à $100 \mathrm{~m}$ de la confluence avec l'Andainette, le site en amont (B) est à $1,8 \mathrm{~km}$, soit 1,5 km des étangs (Figure 1).

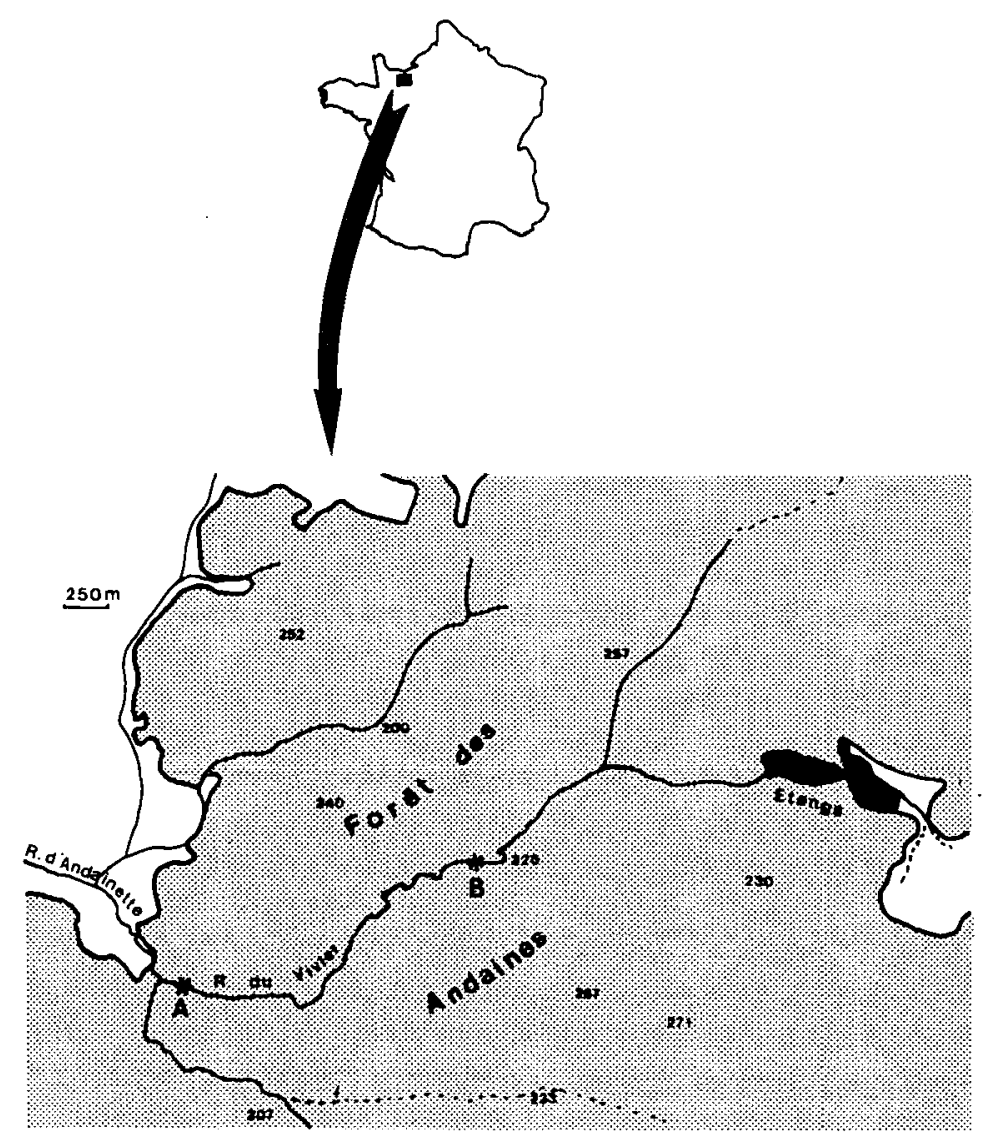

Figure 1

Localisation des sites d'études (A : site aval ; B : site amont).

Figure 1

Localisation of collecting sites (A : downstream site ; B : upstream site). 
Le site $A$ présente une largeur régulière proche de $2 \mathrm{~m}$, la zone d'étude mesure $27 \mathrm{~m}$ de longueur, c'est une zone de rapides en liaison avec une pente forte $(20 \%$ ). L'habitat est donc très caillouteux avec une profondeur moyenne de 10 à $20 \mathrm{~cm}$ en dehors de deux profonds de $40 \mathrm{~cm}$. Les éléments fins (graviers, sables) sont rares, uniquement par petits îlots sous les pierres et quelques poches dans des sous-berges peu profondes. Cette zone est très peu éclairée pendant toute la période d'activité des feuillus : à l'automne les paquets de feuilles sont nombreux mais s'accumulent peu à cause du courant, les détritus sont donc rares y compris dans les profonds.

Le site $B$ en amont est plus irrégulier sa largeur varie de 1,8 à $2,4 \mathrm{~m}$ pour une longueur de secteur de $40 \mathrm{~m}$. La vitesse de courant est plus basse en rapport avec une pente faible $(5 \%$ ). Les habitats sont essentiellement sableux et même vaso-limoneux avec des détritus (branches, feuilles) dans les profonds. Les cailloux sont rares, sauf dans la partie centrale plus rapide, et de taille plus faible qu'en aval, ceux-ci reposent souvent sur des graviers. La profondeur, de 10 à $20 \mathrm{~cm}$ dans les plats (faciès dominant), atteint $40-50 \mathrm{~cm}$ dans deux profonds. Cette zone est bordée d'un côté par des épicéas et de l'autre par quelques gros chênes, ce qui permet un ensoleillement estival partiel contrairement à l'aval. Les pierres sont alors souvent recouvertes par des touffes de Fontinalis sp., les sous berges sont importantes surtout du côté des épicéas.

Les deux sites sont donc différents par leur environnement et la structure de leurs habitats dominants, malgré la faible distance entre eux et un débit comparable du ruisseau. La qualité de l'eau de ces ruisseaux forestiers est bonne et très proche d'un site à l'autre, elle se caractérise par une minéralisation faible et une légère acidité (NEVEU, 1996).

\section{Technique de capture}

Dans l'étude de 1989-1990 deux techniques se sont révélées peu sélectives : la pêche électrique en courant continu et la pêche à la main en remuant le substrat. Compte tenu de la structure du terrain et pour des raisons de facilité, le choix s'est porté sur la pêche à la main de jour.

La pêche s'effectue en progressant de l'aval vers l'amont par 2 à 3 personnes, avec 2 à 3 grandes épuisettes $(60 \times 30 \mathrm{~cm})$ à mailles de $3 \mathrm{~mm}$, disposées face au courant, les unes contre les autres et appuyées sur le fond. Tous les abris potentiels, se trouvant juste en amont des épuisettes sont alors remués, grattés, de façon à déloger les animaux, comme pour certaines études du macrobenthos. Les épuisettes sont toutes relevées, examinées en même temps avant d'être replacées comme précédemment juste en amont de la zone qui a été remuée, puis l'opération recommence. La majorité des individus est ainsi entraînée dans les épuisettes par le courant : si alors quelques écrevisses arrivent à remonter vers l'amont ou nagent transversalement, elles seront délogées à l'étape suivante. II faut environ 2 heures pour échantillonner ainsi une zone d'étude, celle d'aval étant plus complexe par l'abondance des pierres et cailloux. Après capture les écrevisses sont stockées dans des seaux avec l'eau du ruisseau, au niveau de sous secteurs de façon à pouvoir les remettre au mieux dans leur habitat d'origine, ce dernier étant reconstitué par remise en place des abris. Un deuxième passage peut s'avérer nécessaire pour augmenter l'efficacité, qui peut alors atteindre 42 à $65 \%$ de la population en place avec quelques variations selon la structure du substrat (données non publiées). Toutes les pêches effectuées en octobre concernent 2 passages ; celles de juin et août concernent un seul passage et les captures y sont donc plus faibles. 


\section{Méthodes d'analyse}

Chaque individu collecté est mesuré (longueur totale rostre-telson), sexé, éventuellement marqué et remis à l'eau dans son habitat de capture. Pour les femelles leur état de maturité est noté à partir de la coloration des glandes cémentaires ainsi que la présence de spermatophores éventuels, indicatrice de la fécondation.

Les mesures ont été effectuées entre le 10 et 15 octobre de 1990 à 1995 inclus. Cette époque correspond à la fin des mues et représente un stade de référence quant à la stabilisation des structures démographiques. Elle correspond aussi au début des accouplements et permet d'établir le taux de femelles potentiellement reproductrices.

Pour bien définir les limites des classes d'âge un marquage différentiel de groupes a été effectué avec des pêches supplémentaires en juin et août de 1991 à 1993. Le marquage consiste en une coupure droite, avec de petits ciseaux, des bords concaves ciliés des uropodes et/ou du telson. Ces parties se cicatrisent rapidement et le marquage est visible plusieurs mois, même si la partie enlevée est régénérée, sa forme et sa couleur plus claire sont détectables. Un codage simple a été employé, en disposant la queue à plat en vue dorsale, les uropodes sont notés : 1 - 2 - 3 (= telson) - 4 - 5 en partant de la droite vers la gauche. Ainsi le code 1 correspond à une coupure du bord de l'uropode le plus à droite 1 - 3 à la même coupure associée à celle du telson, etc... Pour réduire l'intensité du marquage, le même codage peut être appliqué au même moment à des groupes d'animaux très différents en taille, qui ne risquent pas de se mélanger par la suite.

Pour l'appellation des classes d'âge il est possible d'employer la nomenclature classique sur les poissons : 0+ pour les animaux de l'année en cours, 1+ pour ceux de l'année précédente, etc...

De même pour estimer les variations de la croissance les paramètres classiques sont estimés : taux de croissance $\Delta L=L 1$ - $L 0$, en millimètres ; taux de croissance relatif $\Delta \mathrm{L} / \mathrm{Lo}$, en pour cent de la taille initiale ; taux de croissance annuelle $\Delta \mathrm{L}=\mathrm{Ln}+1-\mathrm{Ln}$ où $\mathrm{n}$ est l'année initiale ; taux de croissance annuel relatif $\Delta \mathrm{L} / \mathrm{Ln}$; taux instantané de croissance pondérale annuel $\mathrm{g}=\log W n+1 / W n, W$ en grammes de poids frais.

Lanalyse statistique repose sur des tests classiques en considérant que la distribution des tailles est proche d'une loi normale : test de $t$ de Student pour la comparaison des moyennes, test de $\mathrm{F}$ de Snedecor pour la comparaison des variances de deux échantillons, test de Bartlett pour la comparaison de variances multiples. Tous ces tests ont été effectués à partir de l'ouvrage de SCHERER (1984) avec un seuil de sécurité de 5 pour cent.

\section{RÉSULTATS}

\section{Structures démographiques}

Les résultats du codage d'octobre 1991 à octobre 1993 permettant de délimiter des groupes d'animaux évoluant parallèlement, cette approche a été effectuée sur le site $A$ en aval où la population est la plus dense.

\section{Caractérisation des classes d'âge du site $A$}

A.partir de la forme des histogrammes la pêche d'octobre 1991 montre (animaux classés de 2 en $2 \mathrm{~mm}$ ) a priori trois grands groupes d'individus : de 20 à $32 \mathrm{~mm}$, de 34 à $52 \mathrm{~mm}$, de 52 à $62 \mathrm{~mm}$. De part et d'autre on retrouve d'une part les juvéniles de l'année entre 13 et $18 \mathrm{~mm}$ et d'autre part quelques individus au-delà de $62 \mathrm{~mm}$ (Figure 2). Le $1^{\text {er }}$ groupe a reçu le code 1 , le $2^{\text {ème }}$ groupe $1-3$, le $3^{\text {ème }}$ groupe $1-5$ et ceux au-delà de $64 \mathrm{~mm}$ 
à nouveau le code 1 . Face à une certaine incertitude dans les limites situées entre le $2^{\text {ème }}$ et le $3^{\text {ème }}$ groupes; : les individus entre 50 et $52 . \mathrm{mm}$-n'ont pas été-codés;; pour la même raison il en est de:même des 62 à $64 \mathrm{~mm}$.

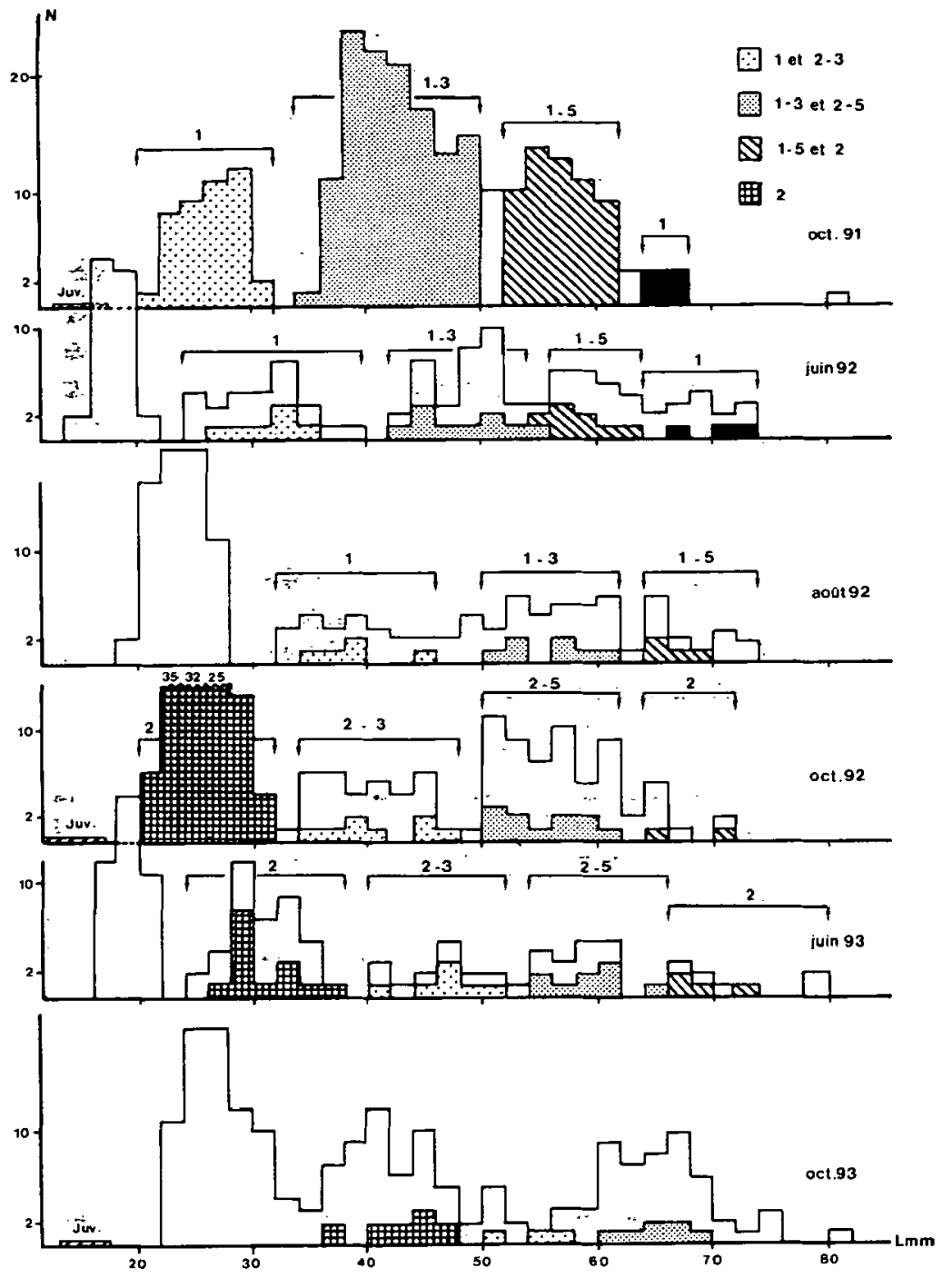

\section{Figure 2}

Structures démographiques en classes de taille des échantillons d'octobre 1991 à octobre 1993: Suivi de l'évolution dans le temps de diveřs groupes d'individus marqués sur lē site A (les zónès hachúrées: corrëspondent aux individus marqués ; leșr flèches indiquent les limites de chäque code-au moment du remarquage. $\mathbf{N}$ : nombre d'individus ; $\mathrm{L}$ : longueur totale en $\mathrm{mm}$; JUV : juvéniles de l'année).

\section{Figure 2}

Size-classes distributions of samples from October 1991 to October 1993. Time evolution of different groups of marked individuals in site A. (shaded areas represent marked individuals; arrows show boundaries of each remarking code ; $\mathrm{N}$ : number of :craytishes ; $\mathrm{L} \cdot:$ individual total length $\mathrm{mm} ; \mathrm{JUV}:$ youngs of the year). 


\section{Tableau I}

Résultats du marquage entre octobre 1991 et 1993 sur le site A : suivi des écrevisses recapturées marquées et du remarquage (11 : intervalles de taille des individus marqués ou remarqués en $\mathrm{mm} ; \mathrm{C}$ : code ; $\mathrm{N}$ : nombre d'individus marqués ou remarqués; 12 : intervalles de tailles des marqués recapturés ; F1 : fréquence des marqués dans les échantillons ; F2 : fréquence des marqués recapturés.

Table I

Marking results between October 1991 and October 1993 in site A. Following of marked-recaptured crayfishes and marking of individuals without mark. (11 : size limits of marked or remarked individuals in millimeters ; $\mathrm{C}$ : code ; $\mathrm{N}$ : number of individuals marked or remarked ; 12 : size limits of marked-recaptured crayfishes ; F1 : frequency of marked individuals in samples ; F2 : frequency of marked-recaptured individuals).

\begin{tabular}{|c|c|c|c|c|c|c|c|c|}
\hline \multirow{3}{*}{ Oct. 91} & 11 & $20-32$ & $34-50$ & & $52-62$ & & $64-68$ & \\
\hline & $\mathrm{C}$ & 1 & $1-3$ & & $1-5$ & & 1 & \\
\hline & $N$ & 59 & 125 & & 57 & & 6 & \\
\hline \multirow{7}{*}{ Juin 92} & $\mathrm{C}$ & & 1 & $1-3$ & & $1-5$ & & 1 \\
\hline & 12 & & $26-36$ & $42-56$ & & $54-64$ & & $66-74$ \\
\hline & $\mathrm{F} 1$ & & 33,3 & 33,3 & & 33,3 & & 25,0 \\
\hline & $\mathrm{F} 2$ & & 11,8 & 8,0 & & 14,0 & & 50,0 \\
\hline & 11 & & $24-40$ & $42-54$ & & $56-64$ & & $64+$ \\
\hline & $C$ & & 1 & $1-3$ & & $1-5$ & & 1 \\
\hline & $N$ & & 27 & 33 & & 21 & & 14 \\
\hline \multirow{7}{*}{$\begin{array}{c}\text { Août } \\
92\end{array}$} & $\mathrm{C}$ & & 1 & & $1-3$ & & $1-5$ & \\
\hline & 12 & & $36-46$ & & $52-62$ & & $64-70$ & \\
\hline & $\mathrm{F} 1$ & & 27,7 & & 26,9 & & 35,7 & \\
\hline & $\mathrm{F} 2$ & & 18,5 & & 21,2 & & 23,8 & \\
\hline & 11 & & $32-46$ & & $50-62$ & & $64 t$ & \\
\hline & $\mathrm{C}$ & & 1 & & $1-3$ & & $1-5$ & \\
\hline & $N$ & & 21 & & 33 & & 14 & \\
\hline \multirow{7}{*}{ Oct. 92} & C & & 1 & & $1-3$ & & $1-5$ & \\
\hline & 12 & & $34-48$ & & $50-62$ & & $64-72$ & \\
\hline & $\mathrm{F} 1$ & & 25,0 & & 20,0 & & 25,0 & \\
\hline & F2 & & 38,0 & & 33,3 & & 14,2 & \\
\hline & 11 & $20-32$ & $34-46$ & & $50-62$ & & $63+$ & \\
\hline & C & 2 & $2-3$ & & $2-5$ & & 2 & \\
\hline & $\mathrm{N}$ & 115 & 31 & & 53 & & 10 & \\
\hline \multirow{7}{*}{ Juin 93} & $\mathrm{C}$ & & 2 & $2-3$ & & $\overline{2-5}$ & & 2 \\
\hline & 12 & & $26-38$ & $40-52$ & & $54-66$ & & $67+$ \\
\hline & $\mathrm{F} 1$ & & 39,4 & 46,6 & & 50 & & 57,1 \\
\hline & $\mathrm{F} 2$ & & 13 & 22,5 & & 16,9 & & 40,0 \\
\hline & 11 & & $24-38$ & $40-52$ & & $54-66$ & & $67+$ \\
\hline & C & & 2 & $2-3$ & & $2-5$ & & 2 \\
\hline & $\mathrm{N}$ & & 40 & 17 & & 18 & & 7 \\
\hline \multirow{4}{*}{ Oct. 93} & $\mathrm{C}$ & & 2 & & $2-3$ & & $2-5$ & \\
\hline & 12 & & $36-48$ & & $50-58$ & & $60-70$ & \\
\hline & $\mathrm{F} 1$ & & 22,9 & & 27,2 & & 17,5 & \\
\hline & $\mathrm{F} 2$ & & 27,5 & & 17,6 & & 38,8 & \\
\hline
\end{tabular}


La pêche suivante en juin 1992 fait apparaître environ 5 groupes à partir de la forme des histogrammes, le code 1 se retrouve au niveau du $2^{\text {eme }}$, le 1-3 dans le $3^{\text {eme }}$, le 1-5 dans le $4^{\mathrm{e} m e}$, recoupant légèrement le précédent, et le 1 dans le petit groupe 5 . Ces résultats se confirment en août 1992, avec disparition des plus grands individus codés. Par ailleurs la croissance des juvéniles de 1991 peut être suivie sans problème particulier puisque le $1^{\text {er }}$ groupe de 14 à $22 \mathrm{~mm}$ en juin, comme son correspondant en août de 18 à $28 \mathrm{~mm}$, est totalement indépendant. A noter aussi que chaque groupe se décale progressivement vers les grandes tailles de la même façon. A chaque remise à l'eau les animaux non codés pour un groupe particulier sont marqués avec le code correspondant.

En octobre 1992 le marquage est changé selon la même procédure avec un code de base 2. Dans tous les cas les limites des classes de longueur définies par les animaux recapturés marqués correspondent bien aux limites que l'on peut définir à partir de la forme des histogrammes et des divers modes visibles. Les structures en octobre sont généralement plus régulières dans la mesure où certaines bimodalités dans un groupe peuvent apparaître en période de mue (juin, août).

A chaque pêche la fréquence des individus marqués de la pêche précédente est relativement élevée : ces fréquences sont un peu plus faibles en octobre après la période de mue (Tableau I).

Le suivi de l'étalement des longueurs à l'intérieur de chaque groupe est possible, surtout à partir des juvéniles de 1991. Ceux ci couvrent une gamme de $6 \mathrm{~mm}$ en octobre 1991, de $8 \mathrm{~mm}$ en juin 1992, de $10 \mathrm{~mm}$ en août 1992, $12 \mathrm{~mm}$ en octobre 1992 et se stabilisent ensuite au niveau des limites déterminées par le retour des individus codés ; en fait en juin 1993 l'étalement est de $14 \mathrm{~mm}$ à partir de la forme de l'histogramme. En considérant le $3^{\text {ème }}$ groupe d'octobre 1991 codé 1-5, celui-ci couvre $10 \mathrm{~mm}$, en juin 1992 encore $14 \mathrm{~mm}$, en août $6 \mathrm{~mm}$, en octobre $8 \mathrm{~mm}$, en juin $19938 \mathrm{~mm}$ et disparaît ensuite au niveau d'animaux codés. Cette contraction progressive du groupe est à relier avec le vieillissement de la cohorte et l'incidence des mortalités. à savoir :

Ces résultats permettent de définir des critères ségrégatifs pour cadrer les cohortes

- les limites des groupes (a priori définis) se conservent au cours du temps ;

- les groupes se déplacent régulièrement dans le temps vers des tailles de plus en plus élevées, de telle manière que le $1^{\text {er }}$ groupe se trouve à la place du $2^{\text {ème }}$ de l'année précédente, on peut donc les considérer comme des cohortes d'animaux semblables et de même âge ;

- l'étalement progressif d'un nouveau groupe confirme les limites acceptables de celui-ci.

L'application de l'ensemble de ces critères permet un découpage en classes d'âge à partir de la forme des histogrammes de chaque année.

\section{Evolution des structures démographiques}

\section{Site A}

L'examen des résultats d'octobre 1990 à octobre 1995 montre que quelques animaux situés à l'intersection de deux distributions sont difficiles à classer. Ils sont placés soit dans l'une, soit dans l'autre selon l'état d'étalement de chacune des cohortes, sinon ils sont répartis par moitié dans chaque classe (Figure $3 A$ ). 

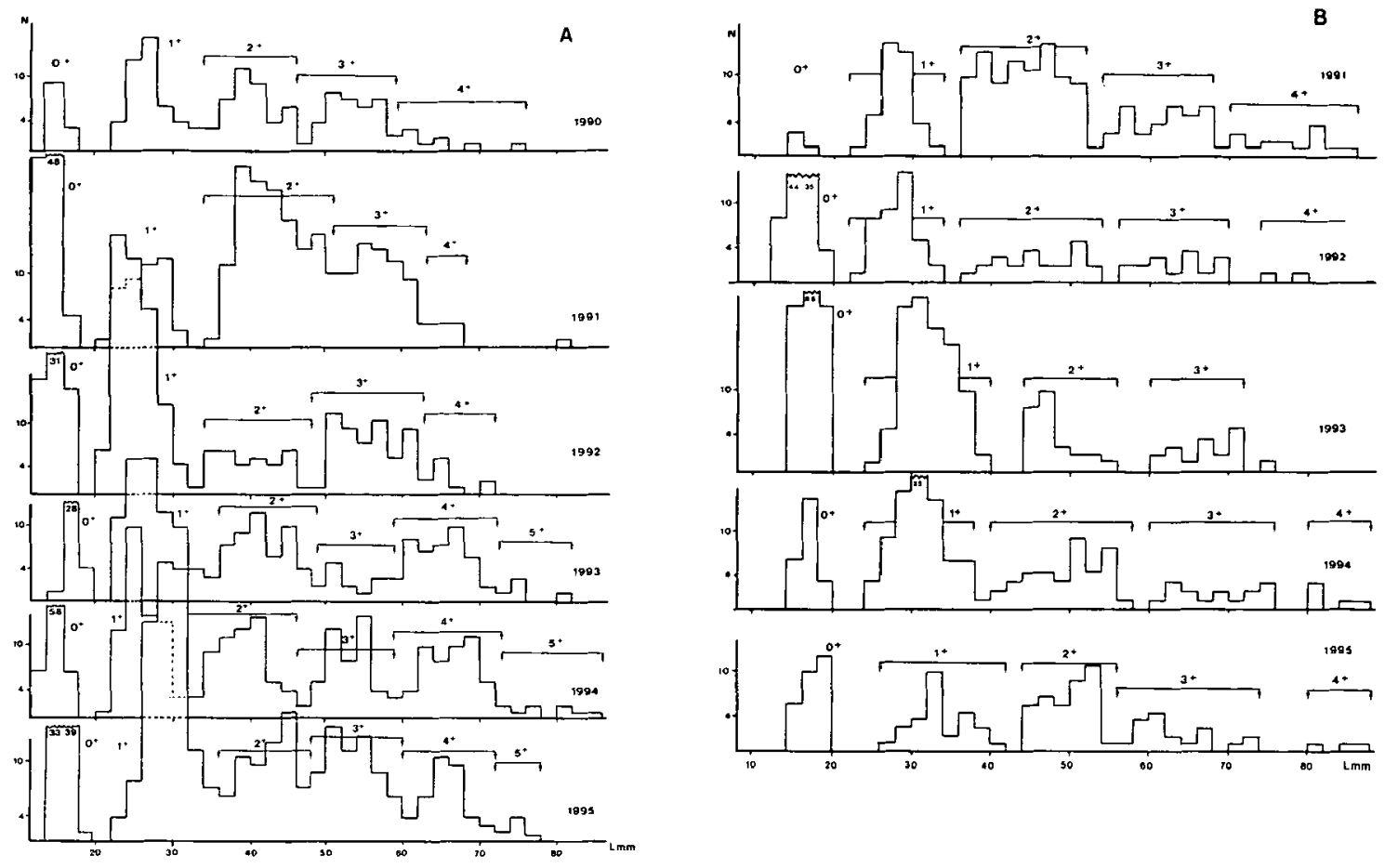

Figure 3

Evolution des structures démographiques en classes de taille mesurées au début d'octobre de 1990 à 1995 sur les 2 sites (les flèches indiquent les limites du découpage en cohortes annuelles. $N$ : nombre d'individus ; $L$ : longueur totale en $\mathrm{mm})$.

\section{Figure 3}

Evolution of size-classes distributions obtained at the beginning of each October month from 1990 to 1995 in the two sites. (arrows show boundaries between annual cohorts ; $N$ : number of crayfishes ; $L$ : individual total length $\mathrm{mm}$ ).

Chaque inventaire montre la présence de 5 à 6 classes d'âge. Ces écrevisses ne vivent pas au-delà de 5 années dans la plupart des cas. Les structures en classes d'âge sont beaucoup plus régulières en 1994 et 1995 que les autres années ; de légers décalages dans la position des 1+ laissent à penser qu'il existe des variations de la croissance entre les années.

\section{Site B}

Ce site n'a pas été échantillonné en octobre 1990. La distribution des classes d'âge est assez différente de l'aval puisque l'étalement des cohortes est plus grand et les zones intercohortales plus marquées (Figure 3B). La séparation entre les 2+ et les 3+ de 1995 a été faite par référence aux années précédentes et par analyse séparée des sexes, la striction entre 54 et $58 \mathrm{~mm}$ est alors plus prononcée puisque les deux individus présents correspondent à 1 mâle et 1 femelle. Par rapport au site A le décalage des âges vers les grandes tailles annonce une croissance plus forte. 


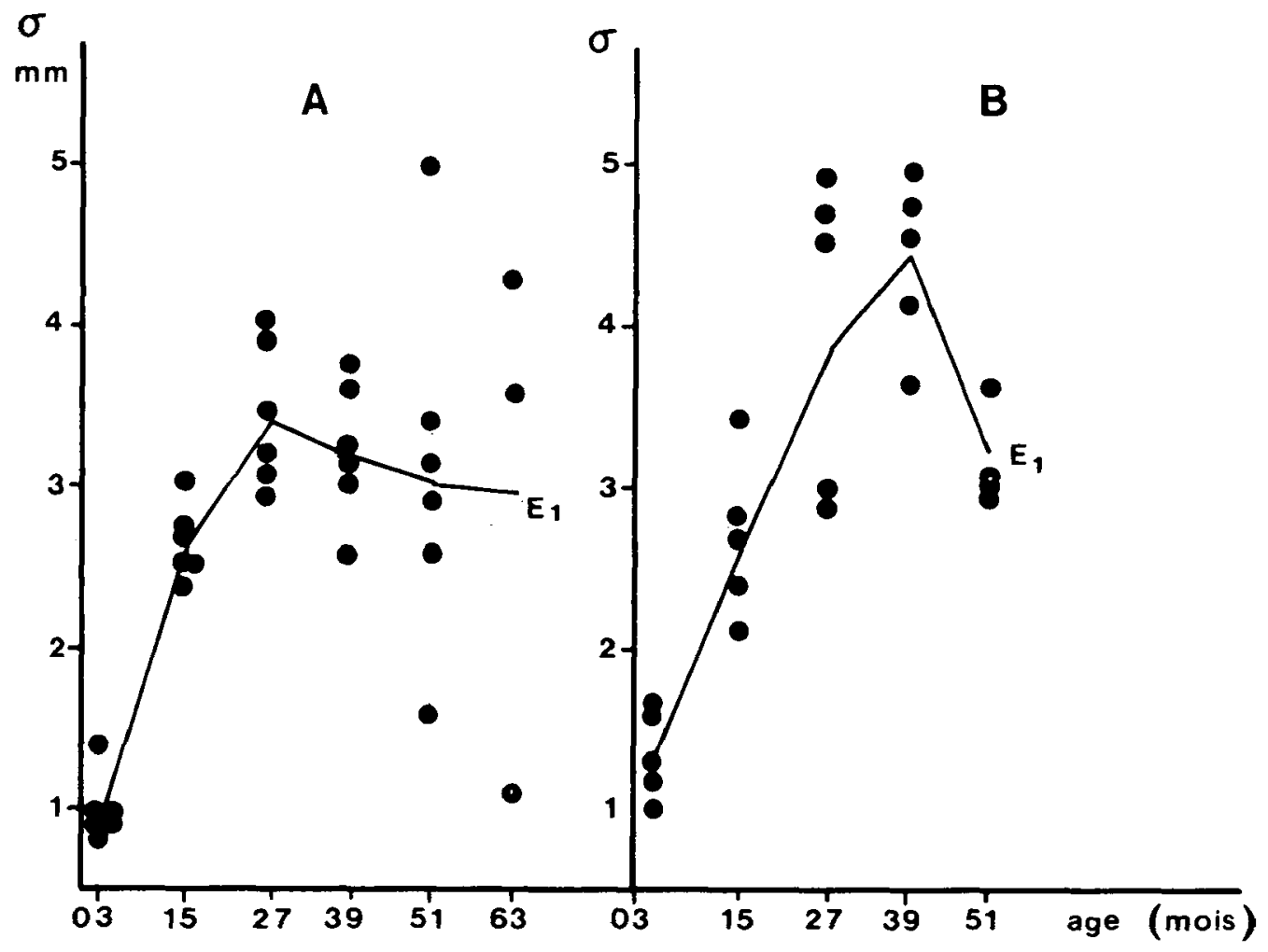

\section{Figure 4}

Variations en fonction de l'âge (mois) de la dispersion des tailles individuelles estimée par l'écart type $(\sigma)$ sur la longueur moyenne (E1 : valeurs moyennes par âge).

\section{Figure 4}

Variations of individual sizes dispersion in relation to crayfishes age, estimated by standard deviation $(\sigma)$ of total length mean (E1 : mean of data per age).

\section{La croissance individuelle}

\section{Validation du découpage cohortal}

Sur le site A l'approche par marquage différentiel de groupes d'animaux ayant une évolution parallèle permet d'établir une première estimation de la taille moyenne individuelle dans chaque groupe et de son évolution temporelle (Figure 2). Les résultats calculés sont présentés dans le Tableau II sous forme de cohortes annuelles avec leur année de naissance (indépendance des juvéniles : fin juin-début juillet).

La croissance est faible entre octobre et juin, d'autant plus que les individus sont âgés. La croissance est plus intense entre juin et août, plus modérée entre août et octobre.

Les mêmes données peuvent être calculées à partir des cohortes définies avec les prélèvements d'octobre de 1990 à 1995 (Figures 3 et 4) et résumées dans le Tableau III. 
La comparaison des chiffres obtenus en aval, en octobre 1992 et octobre 1993, à partir des limites cohortales définies par la recapture d'individus marqués (Tableau II) à ceux obtenus à partir du découpage de l'ensemble des histogrammes (Tableau III), ne donne aucune différence significative entre chaque taille moyenne d'un âge donné (test $t$ ).

\section{Evolution de la taille en fonction de l'âge}

La croissance individuelle est régulière au cours de la vie d'une génération, sur l'ensemble des données du site $A(n=33)$ la longueur moyenne évolue suivant une droite : $L=1,04 t+12,83$, $L$ en $m m$, $t$ en mois depuis l'indépendance, avec $r=0,99$, écart type de la pente $=0,013$, écart type sur l'ordonnée à l'origine $=0,480$. Pour le site $B$ la relation est un peu différente $: L=1,36 t+11,82$ avec $n=24, r=0,99$, écart type de la pente $=0,030$, écart type de l'ordonnée à l'origine $=0,945$. Les individus du site $B$ présentent une croissance plus rapide significativement différente de ceux de $A$ (tests $t$ sur les pentes et les ordonnées à l'origine significatifs). L'ordonnée à l'origine correspond à la taille des juvéniles à leur indépendance. La comparaison des valeurs de $A$ et de $B$ à celle obtenue en mesurant 10 individus (d'une femelle capturée dans un autre ruisseau de la région et mise en élevage), soit $11,50 \mathrm{~mm}$ de moyenne et $\sigma=0,58$, montre que celle de $A$ est significativement plus élevée que cette valeur $(t=5,23)$ contrairement à celle de $B$ $(t=1,02)$.

\section{Tableau II}

Evolution de la taille des individus de chaque cohorte dont les limites sont définies par la présence d'individus marqués entre 1991 et 1993 sur le site A (m : moyenne ; $\sigma$ : écart type de la longueur totale en $\mathrm{mm} ; \mathrm{n}$ : nombre d'individus).

\section{Table II}

Individuals size evolution of each cohort with limits determined by presence of marked individuals, between 1991 and 1993 in site $A$. (m : mean ; $\sigma$ : standard deviation of total length in millimeters ; $\mathbf{n}$ : number of crayfishes).

\begin{tabular}{|c|c|c|c|c|c|c|c|}
\hline \multirow{2}{*}{\multicolumn{2}{|c|}{ COHORTES }} & \multicolumn{6}{|c|}{ DATES } \\
\hline & & $10-91$ & $06-92$ & $08-92$ & $10-92$ & $06-93$ & $10-93$ \\
\hline \multirow{3}{*}{92} & $\mathrm{~m}$ & - & - & - & 15,38 & 19,45 & 27,58 \\
\hline & $\sigma$ & - & - & - & 1,45 & 1,51 & 2,85 \\
\hline & $\mathrm{n}$ & - & - & - & 36 & 41 & 75 \\
\hline \multirow{3}{*}{91} & $\mathrm{~m}$ & 15 & 18,01 & 24,19 & 25,77 & 31,00 & 42,23 \\
\hline & $\sigma$ & 0,96 & 1,77 & 2,14 & 2,44 & 2,66 & 3,18 \\
\hline & $n$ & 33 & 35 & 66 & 115 & 38 & 49 \\
\hline \multirow{3}{*}{90} & $m$ & 26,94 & 31,78 & 39,12 & 40,56 & 46,56 & 53,86 \\
\hline & $\sigma$ & 2,51 & 2,62 & 3,03 & 3,80 & 3,37 & 2,65 \\
\hline & $n$ & 43 & 21 & 16 & 32 & 15 & 11 \\
\hline \multirow{3}{*}{89} & $\mathrm{~m}$ & 43,19 & 49,35 & 56,94 & 56,12 & 59,16 & 65,35 \\
\hline & $\sigma$ & 3,75 & 3,17 & 3,41 & 3,56 & 2,76 & 2,80 \\
\hline & $n$ & 102 & 34 & 29 & 51 & 18 & 40 \\
\hline \multirow{3}{*}{88} & $\mathrm{~m}$ & 57,32 & 59,85 & 66,38 & 67,25 & 69,50 & - \\
\hline & $\sigma$ & 1,68 & 2,45 & 1,45 & 2,71 & 2,30 & - \\
\hline & $n$ & 57 & 22 & 9 & 8 & 7 & \\
\hline \multirow{3}{*}{87} & $\mathrm{~m}$ & 66,5 & 70,57 & - & - & - & - \\
\hline & $\sigma$ & 1,09 & 2,39 & & & & \\
\hline & $n$ & 6 & 13 & & & & \\
\hline
\end{tabular}




\section{Tableau III}

Evolution de la taille des individus de chaque cohorte entre octobre 1990 et octobre 1995 (m : moyenne ; $\sigma$ : écart type de la longueur totale en $\mathrm{mm}$; $\mathrm{n}$ : nombre d'individus ; $N$ : total des captures).

\section{Table III}

Individual size evolution of each cohort between October 1990 and 1995 (m : mean ; $\sigma$ : standard deviation of total length in millimeters ; $n$ : number of crayfishes ; $\mathbf{N}$ : total catches).

\begin{tabular}{|c|c|c|c|c|c|c|c|}
\hline \multirow{2}{*}{$\begin{array}{c}\text { AGE } \\
\text { (mois) }\end{array}$} & & \multicolumn{6}{|c|}{ ANNEES } \\
\hline & & 1990 & 1991 & 1992 & 1993 & 1994 & 1995 \\
\hline \multicolumn{8}{|c|}{ STATION A } \\
\hline & $\mathrm{N}$ & 135 & 276 & 246 & 201 & 264 & 328 \\
\hline \multirow{3}{*}{3} & $\mathrm{~m}$ & 16,08 & 15 & 15,88 & 16,26 & 15,56 & 16,44 \\
\hline & $\sigma$ & 0,90 & 0,96 & 1,45 & 0,88 & 0,80 & 0,96 \\
\hline & $n$ & 12 & 33 & 36 & 15 & 32 & 63 \\
\hline \multirow{3}{*}{15} & $m$ & 27,75 & 26,94 & 25,81 & 27,73 & 26,51 & 29,92 \\
\hline & $\sigma$ & 2,76 & 2,52 & 2,52 & 3,03 & 2,31 & 2,68 \\
\hline & $n$ & 40 & 43 & 115 & 76 & 69 & 96 \\
\hline \multirow{3}{*}{27} & $\mathrm{~m}$ & 40,60 & 43,51 & 40,56 & 42,14 & 39,43 & $42 ; 90$ \\
\hline & $\sigma$ & 2,96 & 4,01 & 3,80 & 3,47 & 3,17 & $3 ; 05$ \\
\hline & $n$ & 40 & 129 & 32 & 50 & 58 & 64 \\
\hline \multirow{3}{*}{39} & $\mathrm{~m}$ & 53,55 & 57,01 & 56,14 & 54,74 & 52,83 & 54,02 \\
\hline & $\sigma$ & 3,16 & 3,15 & 3,75 & 3,62 & 2,55 & 3,06 \\
\hline & $n$ & 34 & 63 & 54 & 12 & 46 & 65 \\
\hline \multirow{3}{*}{51} & $\mathrm{~m}$ & 64,83 & 65,90 & 66,83 & 65,43 & 66,69 & 66,07 \\
\hline & $\sigma$ & 5,00 & 1,60 & 2,82 & 3,19 & 3,39 & 2,54 \\
\hline & $n$ & 9 & 8 & 9 & 43 & 51 & 35 \\
\hline \multirow{3}{*}{63} & $m$ & - & - & - & 76,30 & 79,14 & 75,90 \\
\hline & $\sigma$ & - & - & - & 3,63 & 4,27 & 1,07 \\
\hline & $n$ & - & - & - & 5 & 8 & 5 \\
\hline \multicolumn{8}{|c|}{ STATION B } \\
\hline & $\mathrm{N}$ & & 178 & 133 & 289 & 174 & 119 \\
\hline \multirow{3}{*}{3} & $\mathrm{~m}$ & & 16 & 16,33 & 17,5 & 17,23 & 17,92 \\
\hline & $\sigma$ & & 1 & 1,59 & 1,14 & 1,25 & 1,57 \\
\hline & $n$ & & 4 & 53 & 130 & 23 & 28 \\
\hline \multirow{3}{*}{15} & $\mathrm{~m}$ & & 28,28 & 28,52 & 32,26 & 31,54 & 34,32 \\
\hline & $\sigma$ & & 2,30 & 2,37 & 2,69 & 2,79 & 3,48 \\
\hline & $n$ & & 37 & 39 & 115. & 88 & 29 \\
\hline \multirow{3}{*}{27} & $m$ & & 44,51 & 46,45 & 48,19 & 49,97 & 50,25 \\
\hline & $\sigma$ & & 4,54 & 4,85 & 2,82 & 4,67 & 2,99 \\
\hline & $\mathrm{n}$ & & 89 & 23 & 26 & 42 & 40 \\
\hline \multirow{3}{*}{39} & $\mathrm{~m}$ & & 62,83 & 63,75 & 67,61 & 69,25 & 64,02 \\
\hline & $\sigma$ & & 4,58 & 4,12 & 3,66 & 4,94 & 4,89 \\
\hline & $\mathrm{n}$ & & 36 & 16 & 18 & 16 & 19 \\
\hline \multirow{3}{*}{51} & $m$ & & 79,50 & 77,50 & - & 83,50 & 84,83 \\
\hline & $\sigma$ & & 3,67 & 2,82 & - & 2,82 & 3,05 \\
\hline & $\mathrm{n}$ & & 12 & 2 & - & 5 & 3 \\
\hline
\end{tabular}


Les courbes de croissance peuvent être calculées au niveau de chaque cohorte annuelle (Tableau IV). Quelles que soient les cohortes, l'alignement des points reste important avec des coefficients de corrélation de 0,99, chaque cohorte annuelle présente bien une croissance linéaire à partir des mesures automnales. Pour le site $A$ la pente des droites présente une croissance régulière (modèle exponentiel avec $r=0,87$ pour $n=8$ ) en fonction de la jeunesse de la cohorte, ce qui indiquerait une croissance légèrement plus rapide au début de la vie ; de même l'ordonnée à l'origine se rapproche des mesures expérimentales. Pour le site $B$ l'évolution est plus irrégulière, même si la pente des différentes droites reste plus élevée qu'en $A$, en liaison avec une pression du milieu plus variable.

L'application d'un test $t$ pour comparer les tailles moyennes de chaque âge au niveau des 2 sites donne des résultats significatifs exception faite des $0+$ et des $2+$ de 1991 (Tableau V). La valeur du $\mathrm{t}$ augmente régulièrement avec les années pour chaque âge, ce qui peut se traduire par une augmentation des différences intersites de 1991 à 1994 et par une certaine stabilisation en 1995.

\section{Variabilité des tailles moyennes individuelles}

L'application d'un test $F$ sur les données du site $A$ donne des résultats significatifs pour les classes d'âge 0+, 1+, 2+, 3+ (valeurs respectives du $F: 11,1$ - 27,9 - 15,4 - 15,2), ce qui montre des différences interannuelles dans les tailles moyennes. L'application d'un test $t$ sur les valeurs les plus différentes montre pour les:

- 0+ les tailles de 1993 et 1995 sont plus élevées que celles des autres années;

- 1+ les tailles de 1995, dans une moindre mesure celles de 1990 et 1993 sont supérieures aux autres ;

- 2+ 1991 et 1995 ont des valeurs supérieures ;

- 3+ 1991 et 1992 se distinguent des autres valeurs.

Une année favorable à la croissance pour une classe d'âge ne l'est pas toujours pour les autres, tout dépend de l'histoire individuelle. Mais globalement 1995 a été favorable pour la plupart des individus et dans une moindre mesure 1993 et 1991.

Pour le site $B$ le test $F$ donne des valeurs différentes entre les années pour toutes les classes d'âge $(0+$ à $4+$ ) (valeurs respectives de $F: 12,5-34,2-19,6-7,6-3,6)$. La comparaison des tailles les plus différentes par un test t montre que les tailles des années 1991 et 1992 sont plus faibles que :

- celles des autres années pour les 0+ et les 1+, pour ces derniers 1995 est la meilleure année ;

- celles des années 1994 et 1995, cette dernière étant la meilleure, pour les 2+;

- celles des années 1993 et 1994, cette dernière étant la meilleure, pour les 3+;

- celles des années 1994 et 1995 pour les 4t.

Globalement l'année 1995 est la meilleure année pour le site B, 1991 et 1992 les plus mauvaises, 1993 et 1994 étant intermédiaires.

II y a donc une tendance à une augmentation des tailles au cours des années, plus marquée en amont. 


\section{Tableau IV}

Caractéristiques de la régression linéaire $L=a t+b$ entre la longueur moyenne ( $L: \mathrm{mm}$ ) des individus de chaque cohorte et leur âge ( $t$ : mois) ( $n$ : nombre de classes d'âge ; $r$ : coefficient de corrélation).

\section{Table IV}

Characteristics of linear regression $L=a t+b$ between average length of individuals of each cohort ( $\mathrm{L}: \mathrm{mm})$ and their age ( $\mathrm{t}:$ months) (n : number of age classes ; $r$ : correlation coefficient).

\begin{tabular}{|c|c|c|c|c|}
\hline $\begin{array}{c}\text { ANNEE DE } \\
\text { NAISSANCE }\end{array}$ & $\mathbf{n}$ & $\mathbf{r}$ & $\mathbf{a}$ \\
\hline STATION A & \multicolumn{5}{|c|}{ b } \\
\hline 1987 & 2 & 1 & 1,03 & 13,41 \\
\hline 1988 & 4 & 0,99 & 0,97 & 16,34 \\
\hline 1989 & 5 & 0,99 & 1,04 & 13,82 \\
\hline 1990 & 6 & 0,99 & 1,03 & 12,83 \\
\hline 1991 & 5 & 0,99 & 1,07 & 11,30 \\
\hline 1992 & 4 & 0,99 & 1,06 & 11,80 \\
\hline 1993 & 3 & 0,99 & 1,11 & 11,90 \\
\hline 1994 & 2 & 1 & 1,19 & 11,97 \\
\hline STATION B & & \multicolumn{3}{|l|}{} \\
\hline 1988 & 2 & 1 & 1,28 & 12,05 \\
\hline 1989 & 2 & 1 & 1,56 & 2,65 \\
\hline 1990 & 4 & 0,99 & 1,55 & 5,41 \\
\hline 1991 & 5 & 0,99 & 1,48 & 9,22 \\
\hline 1992 & 4 & 0,99 & 1,34 & 12,50 \\
\hline 1993 & 3 & 0,99 & 1,36 & 12,62 \\
\hline 1994 & 2 & 1 & 1,42 & 12,95 \\
\hline
\end{tabular}

Tableau V

Variations du test de Student au niveau des différences de taille individuelle entre l'amont et l'aval, pour chaque classe d'âge et chaque année (t : $t$ de Student ; $n$ : degré de liberté).

Table V

Variations of Student test on differences of individual size between upstream and downstream sites for each age classe and each year ( $t$ : $t$ Student ; $n$ : degree of freedom).

\begin{tabular}{|c|c|c|c|c|c|c|}
\hline AGE & & 91 & 92 & 93 & 94 & 95 \\
\hline \multirow{2}{*}{$0+$} & $\mathrm{t}$ & 1,96 & 2,86 & 4,07 & 6,04 & 5,52 \\
\cline { 2 - 7 } & $v$ & 35 & 87 & 142 & 53 & 88 \\
\hline \multirow{2}{*}{$1+$} & $\mathrm{t}$ & 2,46 & 5,88 & 10,82 & 12,07 & 3,82 \\
\cline { 2 - 7 } & $\mathrm{v}$ & 78 & 152 & 188 & 155 & 123 \\
\hline \multirow{2}{*}{$2+$} & $\mathrm{t}$ & 1,71 & 5,04 & 7,66 & 13,28 & 9,49 \\
\cline { 2 - 7 } & $\mathrm{v}$ & 216 & 53 & 74 & 98 & 106 \\
\hline \multirow{2}{*}{$3+$} & $\mathrm{t}$ & 7,54 & 6,97 & 9,47 & 17,07 & 9,00 \\
\cline { 2 - 7 } & $v$ & 97 & 68 & 28 & 60 & 81 \\
\hline \multirow{2}{*}{$4+$} & $\mathrm{t}$ & 9,22 & 4,84 & - & 10,70 & 10,83 \\
\cline { 2 - 7 } & $v$ & 17 & 9 & - & 54 & 34 \\
\hline
\end{tabular}


La disparité des tailles individuelles de chaque âge croit avec l'âge quel que soit le site (Figure 4). Cette augmentation est régulière au cours des trois premières années des cohortes (modèle puissance avec pour $A: r=0,967, n=18, y=0,522 x^{0.578}$, y en $\mathrm{mm}, x$ en mois ; pour $\left.B: r=0,920, n=15, y=0,742 \times{ }^{0.491}\right)$. L'application d'un test $F$ sur les données transformées montre que les pentes sont différentes de 0 ( $F=125,2$ pour $A, 45,6$ pour $B$ ). La comparaison des deux courbes montre que les pentes et les ordonnées à l'origine sont différentes (test $t$ : pour les pentes $=19,4$, les ordonnées $=2,73$ ). Le site $B$ présente des tailles plus variables pour les trois premières années de croissance. Pour les animaux plus âgés la variance moyenne se réduit avec l'âge surtout pour le site $A$, il y a donc un tassement des tailles dans chaque cohorte annuelle.

La variabilité interannées des tailles moyennes de chaque cohorte augmente avec l'âge surtout à partir du $2^{\text {eme }}$ été, elle est plus forte en amont, ceci traduit un effet cumulatif des influences du milieu sur l'histoire des individus. Alors que la variabilité relative interannées est légèrement plus forte chez les jeunes individus et pour le site $B$ (Tableau VI). Par ailleurs l'étalement des tailles (exprimé par l'écart type moyen des tailles moyennes individuelles: $\mathrm{E} 1$; les écarts types $\sigma$ du Tableau III étant considérés comme variables aléatoires indépendantes) qui augmente avec l'âge au cours des 3 premières années (Figure 4) est de plus en plus variable avec les années (Tableau VI).

\section{Tableau VI}

Variabilité des estimations de la taille moyenne en fonction des années ( $L$ : moyenne interannées des tailles moyennes individuelles de chaque âge en $\mathrm{mm}$; $\sigma$ : écart type sur cette moyenne ; $n$ : nombre d'années ; CV : coefficient de variation 100 $\mathrm{\sigma} / \mathrm{L}$; $\mathrm{E1}$ : moyenne interannées des écarts types sur la taille individuelle moyenne de chaque âge ; $\sigma 1$ : écart type sur E1; CV1 : coefficient de variation $100 \sigma 1 / E 1)$.

\section{Table VI}

Variability of estimates of average size in relation to years $(L$ : interannual mean of individual average sizes for each age in millimeters ; $\sigma$ : standard deviation ; $\mathrm{n}$ : years number ; $E 1$ : interannual mean of standard deviations on the individual average size of each age ; $\sigma 1$ : standard deviation on E1 ; CV1 : variation coefficient 100o1/ E1).

\begin{tabular}{|c|c|c|c|c|c|c|}
\hline AGE (mois) & 3 & 15 & 27 & 39 & 51 & 63 \\
\hline \multicolumn{7}{|l|}{ STATIONA } \\
\hline $\mathrm{L}$ & 15,78 & 27,44 & 41,52 & 54,71 & 65,95 & 76,98 \\
\hline$\sigma$ & 0,56 & 1,42 & 1,57 & 1,59 & 0,79 & 1,91 \\
\hline$n$ & 6 & 6 & 6 & 6 & 6 & 3 \\
\hline $\mathrm{CV}$ & 3,5 & 5,1 & 3,7 & $2, \overline{9}$ & 1,2 & 2,4 \\
\hline $\mathrm{E} 1$ & 0,99 & 2,63 & 3,41 & 3,21 & 3,05 & 2,89 \\
\hline$\sigma 1$ & 0,23 & 0,24 & 0,42 & 0,43 & 1,12 & 1,69 \\
\hline cV1 & 23,2 & 9,1 & 12,3 & 13,3 & 36,7 & 56,5 \\
\hline \multicolumn{7}{|l|}{ STATION B } \\
\hline $\mathrm{L}$ & 16,99 & 30,98 & 47,87 & 65,49 & 81,33 & \\
\hline$\sigma$ & 0,80 & 2,57 & 2,42 & 2,78 & 3,41 & \\
\hline$n$ & 5 & 5 & 5 & 5 & 4 & \\
\hline $\mathrm{CV}$ & 4,7 & 8,2 & 5,0 & 4,2 & 4,2 & \\
\hline $\mathrm{E} 1$ & 1,31 & 2,72 & 3,97 & 4,43 & 3,09 & \\
\hline o1 & 0,43 & 0,45 & 0,98 & 0,54 & 0,40 & \\
\hline$\overline{c v 1}$ & 32,8 & 16,9 & 24,6 & 11,1 & 12,9 & \\
\hline
\end{tabular}


L'approche de la variabilité des estimations de la taille moyenne peut se faire au niveau des années au lieu des âges, en considérant les variations sur l'ensemble 1+ à 4+, les $0+$ présentant des étalements des tailles trop faibles (Tableau VII). La variance moyenne calculée sur ces 4 classes d'âge reste relativement stable au cours des années pour $A$, mais le coefficient de variation entre les âges se réduit fortement avec les dernières années, en liaison avec une homogénéité de plus en plus grande de l'étalement de chaque cohorte quel que soit l'âge. Par contre l'amont se distingue par une grande hétérogénéité des résultats avec des coefficients de variation interâge relativement élevés mais stables, c'est-à-dire le maintien de différences quant à l'étalement des cohortes entre les âges au cours des années.

\section{Tableau VII}

Variabilité interannuelle moyenne des tailles des individus 1+ à 4+ (E2: moyenne des écarts types de la taille des différents âges de 1+à 4+; $\sigma 2$ : écart type sur E2 ; CV2 : coefficient de variation 100б2/E2).

\section{Table VII}

Interannual average variability of individuals size $1+$ to $4+$ (E2 : standard deviation mean of individuals size $1+$ to $4+; \sigma 2$ : standard deviation ; $n$ : years number ; CV2 : variation coefficient $100 \sigma 2 / E 2)$.

\begin{tabular}{|l|c|c|c|c|c|c|}
\hline & 90 & 91 & 92 & 93 & 94 & 95 \\
\hline \multicolumn{7}{|l|}{ STATION A } \\
\hline $\mathrm{n}$ & 4 & 4 & 4 & 4 & 4 & 4 \\
\hline $\mathrm{E} 2$ & 3,45 & 2,82 & 3,22 & 3,33 & 2,85 & 2,82 \\
\hline$\sigma 2$ & 1,03 & 1,01 & 0,64 & 0,26 & 0,50 & 0,26 \\
\hline cV2 & 29,8 & 35,8 & 19,8 & 7,8 & 17,5 & 9,2 \\
\hline STATION B \\
\hline $\mathrm{n}$ & 4 & 4 & 3 & 3 & 4 & \\
\hline $\mathrm{E} 2$ & 3,75 & 3,54 & 3,05 & 3,80 & 3,60 & \\
\hline o2 & 1,07 & 1,14 & 0,52 & 1,16 & 0,88 & \\
\hline cV2 & 28,5 & 32,2 & 17,0 & 30,5 & 24,4 & \\
\hline
\end{tabular}

\section{Taux de croissance annuel}

L'échantillon plus important du site A entre 1991 et 1993 permet de déterminer le taux de croissance à différentes périodes de l'année (Tableau VIII). L'accroissement maximum s'effectue entre juin et août, la croissance est faible entre août et octobre, la période d'octobre à juin présente une croissance intermédiaire. Les taux relatifs de croissance sont maximums l'été avec 33 à $41 \%$ pour les jeunes individus, ce taux se réduit avec l'âge. Les taux sont comparables pour les 2 années d'étude pour des âges semblables.

Les taux de croissance moyens pour chaque année (vitesse de croissance annuelle) peuvent être calculés sur l'ensemble des données d'octobre 1990 à 1995 et être comparés entre eux puisque établis sur des périodes équivalentes. Ces taux montrent une relative stabilité entre les âges (Tableau IX). Cependant un test $t$ montre que l'accroissement moyen entre 15 et 27 mois en $A$ est différent et plus élevé que ceux en $1+, 4+$ et $5+$, mais ne diffère pas de celui en $3+$ (ce dernier n'atteint pas le seuil de $5 \%$ dans les 
comparaisons avec les valeurs les plus basses). Sur le site B les résultats sont comparables, l'accroissement moyen en $2+$ diffère de ceux en $1+$ et $4+$ (celui à $3+$ n'atteint pas le seuil de signification de $5 \%$, mais seulement $7 \%$ dans les comparaisons avec les valeurs faibles). Globalement l'accroissement en $2+$ et $3+$ représente $45 \%$ de l'accroissement total en $A$ et $55 \%$ en $B$. L'influence de l'année sur la variabilité de l'accroissement moyen n'est pas significative en A (test de Bartlett non significatif) les variances sont semblables, en amont au contraire les variances sont différentes (test de Bartlett : 10,7 donc supérieur à 7,8 valeur limite à $5 \%$ ). Autrement dit en amont la vitesse de croissance annuelle présente une variabilité interannées différente selon les âges.

L'accroissement annuel relatif est important la $1^{\text {ère }}$ année, de 63 à $92 \%$ pour $A$ et 78 à $99 \%$ pour $B$. La décroissance avec l'âge est régulière (site $A: k=-0,158 t+0,866$, t en années, avec $r=0,934$ pour $n=23$; site $B: k=-0,156 t+0,872, r=0,62, n=15$ ), mais les animaux les plus âgés grandissent encore de 13 à $20 \%$ pour la $5^{\text {ème }}$ année.

\section{Tableau VIII}

Taux de croissance et taux de croissance relatif au cours de différentes périodes entre octobre 1991 et octobre 1993 sur le site $A$.

\section{Table VIII}

Growth increments and relative growth increments during different periods between October 1991 and 1993 in site A.

\begin{tabular}{|c|c|c|c|c|c|c|c|c|c|c|c|c|}
\hline \multicolumn{2}{|c|}{$\begin{array}{l}\text { COHORTES } \\
\text { ANNUELLES }\end{array}$} & $10-91$ & & $6-92$ & & $8-92$ & & $10-92$ & & $6-93$ & & $10-93$ \\
\hline \multirow{2}{*}{1992} & $\Delta \mathrm{L}$ & & - & & - & & - & & 4,07 & & 8,13 & \\
\hline & $\mathrm{k}$ & & - & & - & & - & & 0,26 & & 0,41 & \\
\hline \multirow{2}{*}{1991} & $\Delta \mathrm{L}$ & & 3,01 & & 6,18 & & 1,58 & & 5,83 & & 10,63 & \\
\hline & $\mathrm{k}$ & & 0,20 & & 0,34 & & 0,06 & & 0,22 & & 0,33 & \\
\hline \multirow{2}{*}{1990} & $\Delta \mathrm{L}$ & & 4,84 & & 7,34 & & 1,44 & & 6 & & 7,30 & \\
\hline & $k$ & & 0,18 & & 0,23 & & 0,03 & & 0,15 & & 0,15 & \\
\hline \multirow{2}{*}{1989} & $\Delta \mathrm{L}$ & & 6,16 & & 7,59 & & 0 & & 3,04 & & 6,19 & \\
\hline & $\mathrm{k}$ & & 0,14 & & 0,15 & & 0 & & 0,05 & & 0,10 & \\
\hline \multirow{2}{*}{1988} & $\Delta \mathrm{L}$ & & 2,53 & & 6,53 & & 0,97 & & 2,15 & & - & \\
\hline & $k$ & & 0,04 & & 0,11 & & 0,01 & & 0,03 & & - & \\
\hline \multirow{2}{*}{1987} & $\Delta \mathrm{L}$ & & 4,07 & & - & & - & & - & & - & \\
\hline & $k$ & & 0,06 & & - & & - & & - & & - & \\
\hline
\end{tabular}

Sur le site A l'accroissement annuel moyen au cours des 3 premières années de vie présente quelques différences selon les années. Ainsi la croissance au cours de 1992-1993 $(14,28 \mathrm{~mm}, \sigma=1,99)$ et $1994-1995(15,10 \mathrm{~mm}, \sigma=1,11)$ est meilleure qu'en 1993-1994 (10,88 mm, $\sigma=0,74)$, le test $t$ étant significatif (la période 1994-1995 est à la limite du seuil de signification, soit $6 \%$, pour être plus grande que celle de 1991-1992, soit $12,35 \mathrm{~mm}, \sigma=1,42)$.

Sur le site B pour les mêmes âges les différences interannuelles ne sont pas significatives, la vitesse de croissance est plus élevée en amont, mais plus stable au cours des années. 


\section{Tableau IX}

Taux de croissance annuel et taux de croissance relatif annuel au cours de la période 1990-1995 (estimés en octobre de chaque année).

Table IX

Annual growth rate and relative annual growth rate during the period 1990-1995 (estimates at the beginning of October of each year).

\begin{tabular}{|c|c|c|c|c|c|c|}
\hline \multirow{2}{*}{\multicolumn{2}{|c|}{ PERIODE DE MESURE }} & \multicolumn{5}{|c|}{ AGES EN MOIS (ans) } \\
\hline & & $3-15(1+)$ & $15-27(2+)$ & $27-39(3+)$ & $39-51(4+)$ & $51-63(5+)$ \\
\hline \multicolumn{7}{|l|}{ STATION A } \\
\hline \multirow{2}{*}{$1990-91$} & $\Delta$ & 10,86 & 15,76 & 16,40 & 12,35 & 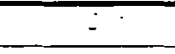 \\
\hline & $\mathrm{k}$ & 0,67 & 0,56 & 0,40 & 0,23 & - \\
\hline \multirow{2}{*}{$1991-92$} & $\Delta$ & 10,81 & 13,62 & 12,03 & 9,82 & - \\
\hline & $k$ & 0,72 & 0,50 & 0,29 & 0,17 & $\therefore$ \\
\hline \multirow{2}{*}{$1992-93$} & $\Delta$ & 12,35 & 16,33 & 14,18 & 9,6 & 9,47 \\
\hline & $k$ & 0,80 & 0,63 & 0,35 & 0,17 & 0,14 \\
\hline \multirow{2}{*}{$1993-94$} & $\Delta$ & 10,25 & 11,70 & 10,69 & 11,95 & 13,40 \\
\hline & $\mathrm{k}$ & 0,63 & 0,42 & 0,25 & 0,22 & 0,20 \\
\hline \multirow{2}{*}{$1994-95$} & $\Delta$ & 14,36 & 16,39 & 14,57 & 13,24 & 9,21 \\
\hline & $\mathrm{k}$ & 0,92 & 0,61 & 0,37 & 0,25 & 0,13 \\
\hline Moyenne & $(\Delta)$ & 11,72 & 14,76 & 13,69 & 11,39 & 10,69 \\
\hline Ecart type & $(\Delta)$ & 1,66 & 2,04 & 2,15 & 1,60 & 2,34 \\
\hline \multicolumn{7}{|l|}{ STATION B } \\
\hline \multirow{2}{*}{$1991-92$} & $\Delta$ & 12,52 & 18,17 & 19,24 & 14,67 & \\
\hline & $k$ & 0,78 & 0,64 & 0,43 & 0,23 & \\
\hline \multirow{2}{*}{$1992-93$} & $\Delta$ & 15,53 & 19,67 & 21,16 & - & \\
\hline & $k$ & 0,95 & 0,69 & 0,45 & - & \\
\hline \multirow{2}{*}{$1993-94$} & $\bar{\Delta}$ & 14,04 & 17,71 & 21,06 & 15,90 & \\
\hline & $k$ & 0,80 & 0,55 & 0,43 & 0,23 & \\
\hline \multirow{2}{*}{$1994-95$} & $\bar{\Delta}$ & 17,09 & 18,71 & 14,05 & 15,58 & \\
\hline & $k$ & 0,99 & 0,59 & 0,28 & 0,22 & \\
\hline Moyenne & $(\Delta)$ & 14,79 & 18,56 & 18,87 & 15,28 & \\
\hline Ecart type & $(\Delta)$ & 1,96 & 0,84 & 3,33 & 0,87 & \\
\hline
\end{tabular}

La vitesse de croissance d'une année peut dépendre de l'histoire des individus et de la croissance acquise. En aval la croissance en $3^{\text {ème }}$ année est reliée linéairement à celle de $2^{\text {ème }}$ année $(r=0,905, n=4)$ celle de $2^{\text {ème }}$ année à celle de $1^{\text {ère }}$ année $(r=0,900, n=4)$. En amont seule la relation entre la croissance en $1^{\text {ère }}$ et $2^{\text {èm }}$ années est significative $(r=0,999, n=3)$. Ainsi l'acquis d'une année relativise celui de l'année suivante, surtout au début de la croissance.

Par contre aucune liaison n'apparaît entre l'importance numérique d'une cohorte et sa vitesse de croissance, ce qui traduit une absence d'effet dépendant de la densité au niveau d'un site. Mais la densité en amont est moindre qu'en aval et pourrait avoir une influence sur la croissance.

Incidence de la taille sur le sex-ratio

En considérant qu'à un âge donné correspond une taille individuelle moyenne relativement stable, il est possible de calculer le rapport des sexes en fonction de la croissance (Tableau X). 
Sur le site A, 621 mâles et 637 femelles ont été capturés, le sex-ratio est équilibré avec $49 \%$ de mâles. Pour le site $B$ les mâles sont un peu moins nombreux que les femelles : 263 contre 349 , soit $43 \%$ des effectifs. Le sex-ratio reste stable en aval, par contre les animaux les plus grands sont surtout des mâles $(77 \%)$, en amont les résultats sont comparables $(65 \%)$. La tendance est à une réduction relative des mâles entre la $1^{\text {ère }}$ et la $3^{\text {ème }}$ années, cette réduction, plus faible en aval, se retrouve en 3+ et 4+.

Au niveau des variations interannées, les mâles sont globalement peu abondants en 1992 en A pour les jeunes individus $(1+$ à $3+)$, cette baisse se retrouve en amont pour les 1+. De même les mâles sont plutôt abondants dans les 4+ que les 3+ en 1991 et 1992 (62 mâles contre 90 femelles), contrairement aux autres années où les femelles sont plus nombreuses.

\section{Tableau $X$}

Evolution du sex-ratio (fréquence des mâles) au cours des années et en fonction des âges.

\section{Table $\mathrm{X}$}

Sex-ratio changes (males frequency) along years and in relation to ages.

\begin{tabular}{|}
\hline ANNEES & \multicolumn{7}{|c|}{ AGE } \\
\hline \multicolumn{7}{|l|}{} & $\mathbf{1 +}$ & $\mathbf{2 +}$ & $\mathbf{3 +}$ & $\mathbf{4 +}$ & $\mathbf{5 +}$ & MOYENNE \\
\hline STATION A \\
\hline 1990 & 0,65 & 0,52 & 0,53 & 0,44 & - & 0,56 \\
\hline 1991 & 0,56 & 0,51 & 0,44 & 0,66 & - & 0,51 \\
\hline 1992 & 0,38 & 0,40 & 0,42 & 0,55 & - & 0,40 \\
\hline 1993 & 0,50 & 0,50 & 0,64 & 0,36 & 0,60 & 0,48 \\
\hline 1994 & 0,47 & 0,50 & 0,52 & 0,39 & 0,87 & 0,48 \\
\hline 1995 & 0,58 & 0,54 & 0,49 & 0,37 & 0,80 & 0,52 \\
\hline Moyenne A & 0,50 & 0,50 & 0,48 & 0,40 & 0,77 & - \\
\hline STATION B & & & & & \\
\hline 1991 & 0,55 & 0,53 & 0,58 & 0,66 & & 0,46 \\
\hline 1992 & 0,35 & 0,52 & 0,25 & 1,00 & & 0,40 \\
\hline 1993 & 0,46 & 0,34 & 0,11 & 1,00 & & 0,40 \\
\hline 1994 & 0,60 & 0,35 & 0,18 & 0,60 & & 0,49 \\
\hline 1995 & 0,38 & 0,41 & 0,38 & 0,33 & & 0,39 \\
\hline Moyenne B & 0,49 & 0,38 & 0,34 & 0,65 & & - \\
\hline
\end{tabular}

\section{Incidence du sexe sur la croissance}

En prenant comme exemple les années 1994 et 1995 où la croissance est meilleure et les cohortes bien caractéristiques, il est possible de comparer les tailles moyennes de chaque sexe pour chaque classe d'âge (Tableau XI).

Généralement la taille des mâles est supérieure à celle des femelles de même âge, mais l'application d'un test $t$ montre que les différences ne sont pas toutes significatives. Ainsi sur le site $A$ la tendance est à une bonne différenciation des tailles sauf exception (1+ de 1994, 2+ et $4+$ de 1995). Par contre en amont aucune différence n'est significative et régulièrement les femelles sont un peu plus grandes. 


\section{Tableau XI}

Différences de croissance entre les mâles et les femelles en octobre 1994 et 1995 ( $\mathrm{m}$ : longueur moyenne en $\mathrm{mm}$; $\sigma$ : écart type ; $\mathrm{n}:$ nombre d'individus ; $\mathbf{d}$ : résultat du test de Student sur la différence des tailles).

\section{Table XI}

Growth differences between males and females during October 1994 and October 1995 ( $m$ : average length of each sex ; $\sigma$ : standard deviation ; $\mathbf{n}:$ number of crayfishes ; $\mathbf{d}$ : results of Student test on size differences).

\begin{tabular}{|c|c|c|c|c|c|c|c|}
\hline \multirow{2}{*}{ AGE } & & \multicolumn{3}{|c|}{1994} & \multicolumn{3}{|c|}{1995} \\
\hline & & Mâles & Femelles & d & Mâles & Femelles & d \\
\hline \multicolumn{8}{|c|}{ STATION A } \\
\hline \multirow{3}{*}{$1+$} & $\mathrm{m}$ & 26,77 & 26,50 & - & 30,39 & 29,00 & +++ \\
\hline & $\sigma$ & 2,54 & 2,41 & & 2,43 & 2,28 & \\
\hline & $n$ & 33 & 36 & & 58 & 64 & \\
\hline \multirow{3}{*}{$2+$} & $\mathrm{m}$ & 40,64 & 38,46 & ++ & 43,08 & 41,96 & $=$ \\
\hline & $\sigma$ & 2,69 & 3,14 & & 3,50 & 3,68 & \\
\hline & $n$ & 28 & 29 & & 34 & 38 & \\
\hline \multirow{3}{*}{$3+$} & $\mathrm{m}$ & 55,41 & 52,45 & ++ & 55,27 & 52,57 & +++ \\
\hline & $\sigma$ & 3,60 & 3,18 & & 2,98 & 2,58 & \\
\hline & $n$ & 29 & 23 & & 35 & 28 & \\
\hline \multirow{3}{*}{$4+$} & $\mathrm{m}$ & 70,07 & 65,62 & +++ & 67,87 & 67,21 & - \\
\hline & $\sigma$ & 3,16 & 2,62 & & 4,91 & 3,49 & \\
\hline & $\mathrm{n}$ & 19 & 31 & & 16 & 14 & \\
\hline \multirow{3}{*}{$5+$} & $\mathrm{m}$ & 82,70 & 74,16 & +++ & - & - & - \\
\hline & $\sigma$ & 1,78 & 2,73 & & & & \\
\hline & $n$ & 5 & 3 & & & & \\
\hline \multicolumn{8}{|c|}{ STATION B } \\
\hline \multirow{3}{*}{$1+$} & $\mathrm{m}$ & 32,33 & 32,77 & - & 34,59 & 34,05 & - \\
\hline & $\sigma$ & 2,96 & 2,57 & & 2,25 & 3,98 & \\
\hline & $n$ & 53 & 35 & & 11 & 18 & \\
\hline \multirow{3}{*}{$2+$} & $\mathrm{m}$ & 51,76 & 49,50 & - & 50,38 & 50,45 & - \\
\hline & $\sigma$ & 3,84 & 5,40 & & 3,08 & 3,29 & \\
\hline & $\mathrm{n}$ & 15 & 27 & & 18 & 23 & \\
\hline \multirow{3}{*}{$3+$} & $\mathrm{m}$ & 66,50 & 69,86 & - & 64,64 & 64,22 & \\
\hline & $\sigma$ & 4,76 & 4,96 & & 5,01 & 4,83 & \\
\hline & $n$ & 3 & 13 & & 7 & 11 & \\
\hline
\end{tabular}

Relation entre la taille et la maturité sexuelle des femelles

Les captures au début d'octobre présentent l'intérêt de porter sur des animaux proches de la reproduction et les femelles mâtures se distinguent par la blancheur des glandes cémentaires. Les femelles fécondées présentent des spermatophores externes.

L'observation précise des individus de 1993 à 1995 montre que la plus petite femelle mâture atteint $54 \mathrm{~mm}$ en aval et $56 \mathrm{~mm}$ en amont (Tableau XII). La taille au-delà de laquelle toutes les femelles sont mâtures est de $56 \mathrm{~mm}$ en aval, $58 \mathrm{~mm}$ en amont. II y a une légère tendance à être mature à une taille un peu faible en aval (seuil de signification $16 \%$ ) en relation avec une croissance plus faible. 
De même la fréquence des femelles matures par rapport à l'ensemble des femelles (supérieures à $20 \mathrm{~mm}$ ) est plus élevée en aval : 15,3 à $31,7 \%$ contre 11,7 à $16,8 \%$. Mais c'est l'inverse en ce qui concerne la fréquence des femelles en âge de procréer $(66,6$ à $86,6 \%$ en aval contre 68,7 à $100 \%$ en amont).

Globalement l'état de maturité est meilleur en 1994 dans les deux sites, mais la comparaison interannées reste limitée faute d'un échantillonnage suffisant. De même les accouplements n'ont pas commencé au début octobre 1995 contrairement aux deux autres années, mais dans tous les cas il s'agit du début des accouplements. Dans l'étude préliminaire de $1989-1990$, début janvier $78 \%$ de la population de femelles potentiellement mâtures portent des œufs.

\section{Tableau XII}

Caractéristiques des femelles reproductrices au début d'octobre de 1993 à 1995 (Lo : taille minimum à la maturité ; L1 : taille au-delà de laquelle toutes les femelles sont mâtures ; Fo : fréquence des femelles fécondées ; F1 : fréquence des femelles matures par rapport à l'ensemble des femelles supérieures à $20 \mathrm{~mm}$ de longueur ; F2 : fréquence des femelles matures par rapport à l'ensemble des femelles supérieures à Lo).

\section{Table XII}

Matured females characteristics at October beginning from 1993 to 1995 (Lo : minimum size of sexual maturity ; L1 : size beyond which all females are mature ; Fo : fertilized females frequency ; F1 : matured females frequency in relation to all females above. $20 \mathrm{~mm}$ length ; F2 : matured females frequency in relation to all females above Lo).

\begin{tabular}{|c|c|c|c|c|c|}
\hline & L0 & L1 & F0 & F1 & F2 \\
\hline STATION A \\
\hline 1993 & 56 & 64 & 2,6 & 26,8 & 86,6 \\
\hline 1994 & 54 & 56 & 4,8 & 31,7 & 81,2 \\
\hline 1995 & 56 & 58 & 0 & 15,3 & 66,6 \\
\hline STATION B & 58 & 60 & 2,1 & 11,7 & 68,7 \\
\hline 1993 & 56 & 58 & 1,6 & 16,8 & 100 \\
\hline 1994 & 62 & 62 & 0 & 12,0 & 100 \\
\hline 1995 &
\end{tabular}

\section{DISCUSSION ET CONCLUSIONS}

L'étude sur.6 années successives d'une population d'Austropotamobius pallipes apporte des résultats sur l'évolution à moyen terme de quelques paramètres démographiques. Les études sur plusieurs années sont peu nombreuses et il est difficile d'effectuer des comparaisons sur la variabilité. Les rares données concernent l'évolution de la croissance de cette espèce au cours de 2 ans par PRATTEN (1980) et sur 3 ans par BREWIS et BOWLER (1982) (le premier dans le Sud de l'Angleterre, les seconds dans le Nord). En France FENOUIL (1987) a étudié 2 ruisseaux de Provence sur 2 ans, mais avec un suivi plus irrégulier que dans les études anglaises, par contre les 2 ruisseaux présentent des croissances différentes.

Le principal problème .rencontré pour ces études démographiques repose sur l'efficacité de l'échantillonnage et la caractérisation des classes d'âge. Les techniques de captures sont souvent très sélectives (NEVEU, 1996) et beaucoup de travaux négligent ce 
biais, en particulier au niveau des jeunes individus. Le choix de la pêche à la main de jour avec des épuisettes à mailles fines pallie cet inconvénient. Les méthodes d'analyse des structures polymodales, méthodes indirectes faute de critères d'âge, sont souvent employées pour définir les classes d'âge sans tenir compte des conditions d'application et sans références biologiques, le découpage peut être ambigu (Mc DONALD et PITCHER, 1979). C'est surtout la taille minimum des échantillons qui n'est pas respectée. L'approche biologique, in situ par marquage (BREWIS et BOWLER, 1982), éventuellement expérimentale pour compter les mues (PRATTEN, 1980 ; FENOUIL, 1987), permet de suivre l'évolution des cohortes ou de les reconstituer.

En Normandie des données préliminaires ont montré une relative ségrégation visuelle des âges sur des histogrammes de fréquences de taille ; dans plusieurs ruisseaux les plus grands échantillons ont pu être découpés par la méthode de BHATTACHARYA (1967). Dans l'étude présente, l'objectif a été d'aborder des populations de densités moyennes où les échantillons varient de 119 à 328 individus, c'est-à-dire à la limite de validité des approches d'extraction polymodale selon FRANCE et al. (1991). Pour. cela le suivi par marquage différentiel-de groupes d'individus a priori monocohortaux, permet de suivre leur évolution temporelle. II s'avère que ces groupes restent homogènes, les individus évoluent d'une manière synchrone et après chaque année une cohorte donnée se positionne à la place de la suivante. Le taux de recapture des animaux marqués est assez important pour définir les limites intergroupes. Par ailleurs l'étalement progressif des cohortes peut être suivi au début de la croissance, la cohorte des juvéniles et des $1+$ reste indépendante jusqu'à la fin du $2^{\text {eme }}$ été, le marquage cohortal montre aussi qu'à partir de cette période la diversité des tailles individuelles intracohortale reste relativement-stable. Ce critère apporte des éléments supplémentaires quant à la réalité du découpage en classes d'âge à partir des structures en classes de taille. En relation avec une.croissance meilleure, les cohortes de l'amont sont plus individualisées malgré un étalement un peu plus élevé, ce qui est l'inverse de l'évolution longitudinale de la:croissance dans d'autres ruisseaux de la région (NEVEU, op. cit.).

II est possible de s'interroger sur l'incidence éventuelle. du marquage sur la croissance, suite aux remarques de GUAN.(1997)-qui. note une réduction de croissance chez Pacifastacus leniusculus. Or la comparaison (test $t$ ). des moyennes des tailles des individus marqués et celles des non marqués, pour un même âge, ne donne pas de différences significatives.

La croissance en longueur, mesurée à chaque automne, est très régulière et linéaire au cours des 5 à 6 ans de vie de la grande majorité des individus. Cette linéarité reste constante d'une cohorte à l'autre, avec une légère évolution dans la pente dans le site aval, traduisant une croissance un peu plus rapide des jeunes cohortes. L'ordonnée à l'origine de ces modèles donne une bonne estimation de la taille à l'émancipation qui correspond aux mesures directes et est proche de celle trouvée par FENOUIL (1987) dans des ruisseaux du Sud de la France. L'estimation de cette. taille: est plus élevée en aval, il est possible d'émettre plusieurs hypothèses:

- la taille des. œufs est plus élevée en aval. L'explication pourrait être qu'une croissance plus rapide des femelles : en amont réduit la taille des œufs et inversement, associée à une influence de l'âge des femelles:(plus jeunes en amont) ;

- le temps calculé depuis l'indépendance réelle est de 3 mois en amont (juillet à octobre), mais celui en aval serait de. 4 mois (ce qui donne $L O=11,79$, semblable aux données directes) àlors que le régime thermique est plus bas ce qui est en contradiction avec des éclosions.plus précoces ;

- le décalage des périodes d'accouplement et de ponte, cela·ne semble pas être le cas d'après le Tableau XII. 
II faudrait des mesures complémentaires pour vérifier ces différentes hypothèses, mais il est à noter que les juvéniles de FENOUIL (1987) ne mesurent que $11 \mathrm{~mm}$ à l'indépendance, donc sont un peu plus petits que ceux mesurés directement en Normandie $(11,50 \mathrm{~mm})$. Ces résultats correspondent à des animaux ayant un développement embryonnaire plus rapide en raison du niveau thermique de ces ruisseaux de Provence, ce qui correspondrait au même phénomène sur le ruisseau du Vivier pour la partie amont.

La taille moyenne de chaque âge est supérieure en amont et la tendance augmente chaque année de 1991 à 1994, en 1995 la croissance augmente aussi en aval. Ces différences sont du même ordre de grandeur que celles (différence de 16 à $17 \mathrm{~mm}$ à 3 ans) trouvées entre des ruisseaux ayant des niveaux thermiques différents en Provence par FENOUIL (op. cit.) et par ROQUEPLO (1997) pour des ruisseaux du Pays Basque, des Cévennes et de la Corrèze. Les tailles atteintes pour chaque âge sont proches de celles de PRATTEN (1980) pour le Sud de l'Angleterre et de celles de ZEKHNINI et CHAISEMARTIN (1991) dans le Limousin. Même si aucun enregistrement continu de la température n'a été effectué sur les deux sites, des mesures ponctuelles montrent des différences entre 1 et $3^{\circ} \mathrm{C}$ en été, les maxima étant plus élevés en amont.

L'accroissement relatif de la taille diminue régulièrement avec l'âge quel que soit le site ou l'année, cette décroissance a été notée aussi par PRATTEN (1980), puis BREWIS et BOWLER (1982), ainsi qu'une augmentation de cette vitesse de croissance chez les juvéniles, puis une stabilisation chez les subadultes et enfin une réduction chez les adultes. Ces résultats se retrouvent aussi dans les observations de FENOUIL (1987). En Normandie l'évolution est comparable, mais la croissance reste minimale et relativement stable chez les adultes. Cette évolution peut aussi être comparée au niveau du taux instantané de croissance pondérale, la conversion en masse (gr) s'effectue avec les équations de l'étude préliminaire (NEVEU, 1996). Pour la période allant du mois d'octobre de l'année de naissance au mois d'octobre de l'année suivante, $g=1,70$ en aval et 1,84 en amont ; pour l'année suivante $\mathrm{g}=1,27$ en aval, 1,34 en amont. Pour les mêmes périodes BREWIS et BOWLER (op. cit.) trouvent en moyenne 0,97 et 0,92 pour le Nord de l'Angleterre et PRATTEN (op. cit.) 3,03 et 1,71 pour le Sud. Les valeurs' de Normandie sont donc intermédiaires.

L'application des résultats de FENOUIL (op. cit.) au niveau du nombre de mues, montre (hypothèse : 1 mue $=+2 \mathrm{~mm}$ la tère année, $+3 \mathrm{~mm}$ la $2^{\text {ème }}$ et $3^{\text {ème }}$ et $+4 \mathrm{~mm}$ ensuite) que pour la Normandie la moyenne est de 2,4 mues en aval et 2,9 mues en amont pour la période juillet-octobre de l'année de naissance, 3,9 en aval et 5,7 en amont pour l'année suivante, 4,9 et 6,2 pour la $3^{\text {àme }}$ année, 3,4 et 3,5 pour la $4^{\text {ème }}$ année, 2,8 et 3,8 pour la $5^{\text {ème }}$ année et 2,6 pour la $6^{\text {ème }}$ en aval. Ces résultats sont un peu plus élevés que ceux obtenus en Angleterre.

Les variations de croissance au cours des années montrent que les années 1991 et 1992 sont les moins favorables, en effet les hivers 1990-1991 et 1991-1992 ont été plus longs. Des mesures effectuées dans la région de Rennes $( \pm 100 \mathrm{~km})$ donnent 4 à 6 semaines inférieures à $5^{\circ} \mathrm{C}$ et 17 à 18 semaines inférieures à $10^{\circ} \mathrm{C}$ pour ces hivers, contre seulement 1 semaine inférieure à $5^{\circ} \mathrm{C}$ et 8 inférieures à $10^{\circ} \mathrm{C}$ pour l'hiver $1994-1995$ (mesures moyennes effectuées à $1 \mathrm{~m}$ de profondeur dans divers plans d'eau).

Au début de la vie d'une cohorte la variabilité des tailles augmente avec l'âge, elle se stabilise ensuite, alors que dans l'étude de MANKAMPA (1995) effectuée dans le Limousin, elle décroît. Cette synchronisation plus grande peut être reliée à un nombre de mues plus faible qui réduit les différences interindividus. La variabilité reste plus élevée en amont. Les différences d'étalement de chaque cohorte annuelle se réduisent avec l'âge surtout en aval, c'est-à-dire que les variations interannées de la pression du milieu se font de moins en moins sentir. 
La période de croissance effective au cours d'une année se réduit à la période estivale et une partie du printemps : dès le mois d'août il y a un fort ralentissement des mues (pour le Sud de l'Angleterre PRATTEN (1980) parle de juin à octobre). Au cours de la vie la croissance maximum s'effectue au cours de la $2^{\text {ème }}$ et $3^{\text {ème }}$ années $( \pm 50 \%$ de l'acquis total). La vitesse de croissance est plus élevée en amont, mais plus variable selon les années. C'est l'inverse en aval où la forêt dense réduit la croissance, mais a un effet tampon sur sa variabilité (effet thermique).

La croissance en $2^{\text {ème }}$ année dépend du niveau acquis en $1^{\text {ère }}$ année et plus la taille est forte plus la croissance en $2^{\text {ème }}$ année sera faible, ce phénomène se retrouve en $3^{\text {ème }}$ année, mais est beaucoup moins net. Cette régulation a tendance à homogénéiser les structures démographiques au moment de la maturité des gonades et permet de réduire les interactions.

Le sex-ratio est équilibré en aval, un peu moins en amont, les mâles sont relativement moins nombreux avec l'âge, en particulier au moment de la maturité, mais ils ont tendance à vivre plus vieux, probablement en liaison avec une surmortalité des femelles après 1 ou 2 reproductions. La taille moyenne des mâles a tendance, au moins en aval, à être supérieure à celles des femelles (à âge égal). De nombreux auteurs trouvent des différences de croissance entre les sexes (BREWIS et BOWLER, 1982 ; NEVEU, 1996 ; BERNARDO et al., 1997 ; ROQUEPLO, 1997) par contre d'autres ne trouvent pas de différence (PRATTEN, 1980 ; ZEKHNINI et CHAISEMARTIN, 1991 ; MANKAMPA, 1995) ou trouvent les 2 cas (ZEKHNINI et CHAISEMARTIN, op. cit.).

La maturité sexuelle débute la $3^{\text {ème }}$ année chez les femelles, elle est totale en $4^{\text {ème }}$ année, ce qui correspond à la plupart des résultats obtenus en France et en Angleterre, de même en ce qui concerne la taille à la $1^{\text {ère }}$ reproduction. Mais les femelles ne vieillissent guère plus et en fait la majorité se reproduit 1 à 2 fois seulement. La période des accouplements dépend du climat de l'année, mais débute généralement la $1^{\text {ere }}$ quinzaine d'octobre, sauf en 1995 où la douceur de l'année recule l'échéance. Cette période est plus précoce qu'en Provence (FENOUIL, op. cit.) mais avec une indépendance plus tardive des juvéniles en rapport avec un niveau thermique plus bas. Pour le Portugal BERNADO et al. (1997) trouvent une maturité à la même taille, mais qui est atteinte à 2 ans.

L'étude régionale de 1989-1990 montrait une relation étroite entre la croissance et la distance à la source, ce qui a priori est corrélée à l'évolution de la température, normalement de plus en plus élevée vers l'aval. Les enregistrements des températures au cours de l'été 1990 montrent en effet une différence thermique importante et progressive, surtout au niveau des maxima, sur la rivière Cance (NEVEU, 1996). Dans le cas du ruisseau du Vivier cette "logique » longitudinale est inversée par la structure du milieu et/ou la présence des étangs. Si l'ensemble de cette variabilité de la croissance est prise en compte, y compris sur l'évolution au cours des 6 années, l'écrevisse à pattes blanches pourrait être considérée comme un bon bioindicateur de l'évolution thermique locale et régionale. A partir d'études à long terme standardisées peut être pourrait-elle être un témoin du changement climatique global.

Au niveau de la qualité de l'eau aucune différence ne permet de distinguer les deux sites, en liaison semble-t-il avec le rôle tampon de la forêt. De même, au niveau sanitaire, quelques rares individus sont atteints de thélohaniose, mais à un taux beaucoup plus faible que sur la Cance (NEVEU, op. cit.). Par contre, il est possible de s'interroger sur l'éventualité de l'Aphanomycose compte tenu de la présence de Pacifastacus leniusculus dans la région, cette espèce étant un remarquable réservoir (NEVEU, 1998a et b). D'autant que les zoospores de ce champignon peuvent survivre dans le mucus des poissons (truites), en particulier les allochtones qui servent au repeuplement. Ce risque s'est peut être concrétiser en 1995-1996 avec la disparition de la population dans la zone d'étude et les ruisseaux de la région, ce qui n'a pas permis de poursuivre les mesures sur un plus 
long terme. Mais une autre hypothèse peut être émise sur ces mortalités en liaison avec les observations faites en 1990 sur la rivière Cance. Au cours de l'été 1990, particulièrement chaud (maxima de l'eau autour de $24-25^{\circ}$ en aval) les écrevisses ont commencé à mourir fin juillet, les mortalités ont progressées vers l'amont jusqu'en novembre, aucune trace de pathogènes n'a été décelée jusqu'à la disparition totale du stock (données non publiées).

Or l'année 1995 a été particulièrement chaude et l'accélération de la croissance, comme la réduction de sa variabilité étaient peut-être les prémices d'un déséquilibre écophysiologique pouvant avoir à terme des conséquences sur la survie. Mais dans cette hypothèse les mortalités auraient eu lieu au cours de l'été-automne, soit 1995, soit 1996 comme sur la Cance en 1990, avec des individus " malades " et/ou des exosquelettes visibles en octobre. Ce ne fut pas le cas, ni à l'automne 1995, ni en 1996.

En conclusion, cette étude montre la variabilité des populations dans un petit ruisseau sur une distance faible, malgré un couvert forestier dense et ancien qui aurait pu faire croire a priori à une forte stabilité environnementale. En comparant globalement les 2 sites, la partie aval, très boisée, plus fraîche, à pente plus forte, donne une croissance plus lente, mais plus régulière dans le temps. La partie amont, avec une croissance meilleure, mais plus instable, est probablement soumise non seulement à des variations thermiques plus fortes sous l'influence des étangs d'où est issu le ruisseau, mais aussi à l'influence de conditions trophiques différentes. Ceci est lié d'une part à d'éventuels apports des étangs, d'autre part à un ensoleillement plus fort du site (périphyton plus abondant, Fontinalis) et à l'accumulation de détritus en voie de décomposition (feuilles) présents toute l'année.

Autrement dit la vérification des hypothèses d'homéostasie des populations locales de l'étude régionale de 1989-1990 (NEVEU, 1996) avait aussi besoin d'un minimum de contraste résultant d'une association minimum entre stabilité et variabilité partielles au niveau stationnel.

\section{BIBLIOGRAPHIE}

BERNADO J.M., ILHEN M., COSTA A.M., 1997. Distribution, population structure and conservation of Austropotamobius pallipes in Portugal. Bull. Fr. Pêche Piscic., 347, 617-624.

BHATTACHARYA C.G., 1967. A simple method of resolution in gaussian components. Biometrics, 23, 115-135.

BREWIS J.M., BOWLER K., 1982. The growth of the freshwater crayfish Austropotamobius pallipes in Northumbria. Freshwater Biology, 12, 187-200.

DAVIES I.J., 1989. Population collapse of the crayfish Orconectes virilis in response to experimental whole lake acidification. Can. J. Fish Aquat. Sci., 46, 910-922.

FENOUIL E., 1987. Biologie et développement de l'écrevisse Austropotamobius pallipes pallipes (Lereboullet, 1858) en région provençale. Thèse Doct. es Sciences, Univ. Aix Marseille III, $291 \mathrm{p}$.

FRANCE R., HOLMES J., LYNCH A., 1991. Use of size frequency data to estimate the age composition of crayfish populations. Can. J. Fish Aquat. Sci., 48, 2324-2332.

GUAN R.Z., 1997. An improved method for marking crayfish. Crustaceana, 70, 6, 641-652.

MANKAMPA M., 1995. Approche de la biodiversité démographique, trophique et nutritionnelle de 5 espèces d'écrevisse dans un secteur géographique Limousin. Thèse Doct. Univ. Limoges, $191 \mathrm{p}$. 
MC DONALD P.D.M., PITCHER T.J., 1979. Age-groups from size-frequency data : a versatile and efficient method of analysing distribution mixtures. J. Fish. Res. Board Can., 36, 987-1001.

NEVEU A., 1996. Caractéristiques démographiques de stocks résiduels de l'écrevisse à pattes blanches, Austropotamobius pallipes (Astacidae), en Normandie. Cybium, 20, 3, 75-93.

NEVEU A., 1998a. Présence de l'aphanomycose en France : suivi d'un foyer dans l'Ouest de 1990 à 1998. L'astaciculteur de France, 57, 2-5.

NEVEU A., 1998b. Pacifastacus leniusculus : son rôle de vecteur et de réservoir de la peste des écrevisses (Aphanomycose). Etat actuel des connaissances. L'astaciculteur de France, 57, 6-11.

PRATTEN D.J., 1980. Growth in the crayfish Austropotamobius pallipes (crustacea : Astacidae). Freshwater Biology, 10, 401-412.

ROQUEPLO C., 1997. Etude de la population d'écrevisses à pattes blanches (Austropotamobius pallipes, Lereboullet) en cours d'eau, soumises à une pêche de loisir. L'astaciculteur de France, 51, 59-70.

SCHERER B. 1984. Biostatistiques. MORIN G. (ed.), Québec, 850 p.

ZEKHNINI A., CHAISEMARTIN C., 1991. Structure démographique et éthologie alimentaire de l'écrevisse pallipède (Austropotamobius pallipes) dans quatre cours d'eau. Vie Milieu, 41, 1, 45-53. 